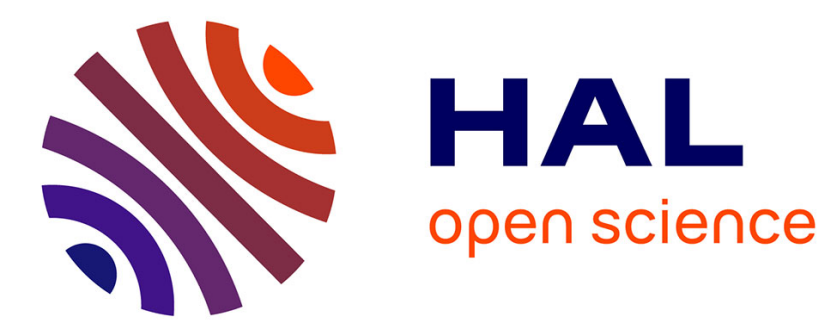

\title{
Decision Making with Imprecise Probabilistic Information
}

Thibault Gajdos, Jean-Marc Tallon, Jean-Christophe Vergnaud

\section{To cite this version:}

Thibault Gajdos, Jean-Marc Tallon, Jean-Christophe Vergnaud. Decision Making with Imprecise Probabilistic Information. Journal of Mathematical Economics, 2004, 40 (6), pp.647-681. 10.1016/j.jmateco.2003.06.004 . halshs-00086021

\section{HAL Id: halshs-00086021 https://shs.hal.science/halshs-00086021}

Submitted on 17 Jul 2006

HAL is a multi-disciplinary open access archive for the deposit and dissemination of scientific research documents, whether they are published or not. The documents may come from teaching and research institutions in France or abroad, or from public or private research centers.
L'archive ouverte pluridisciplinaire HAL, est destinée au dépôt et à la diffusion de documents scientifiques de niveau recherche, publiés ou non, émanant des établissements d'enseignement et de recherche français ou étrangers, des laboratoires publics ou privés. 


\title{
Decision Making with Imprecise Probabilistic Information *
}

\author{
Thibault Gajdos $†$ Jean-Marc Tallon $\ddagger$ Jean-Christophe Vergnaud ${ }^{\S}$
}

June 2002. Revised May 2003.

\begin{abstract}
We develop an axiomatic approach to decision under uncertainty that explicitly takes into account the information available to the decision maker. The information is described by a set of priors and a reference prior. We define a notion of imprecision for this informational setting and show that a decision maker who is averse to information imprecision maximizes the minimum expected utility computed with respect to a subset of the set of initially given priors. The extent to which this set is reduced can be seen as a measure of imprecision aversion. This approach thus allows a lot of flexibility in modelling the decision maker attitude towards imprecision. In contrast, applying Gilboa and Schmeidler (1989) maxmin criterion to the initial set of priors amounts to assuming extreme pessimism.
\end{abstract}

Keywords: Uncertainty, Decision, Multiple Priors.

JEL Number: D81.

\section{Introduction}

Ellsberg (1961) experiments have established in a convincing and robust way that decision makers generally prefer to act in settings in which they have better information. In the classic two-urn experiments, subjects tend to choose to bet on the color of a ball drawn from an urn whose composition is known, say, 50 black balls and 50 white balls rather than on the color of a ball drawn from an urn that contains black and white balls in unknown proportion. These experiments have led to a host of models of decision under uncertainty, such as the Choquet expected utility model (Schmeidler (1989)) or the multiple prior model (Gilboa and Schmeidler (1989)), capturing the fact that agents might have aversion towards uncertainty. However, these models, while referring to the Ellsberg's experiments neglect an important aspect of these experiments: the information the decision maker has when making his choice is not part of the modelling.

\footnotetext{
${ }^{*}$ We thank M. Cohen, J.Y. Jaffray, M. Scarsini and B. Walliser for useful discussions and comments. The comments of a referee were very helpful to clarify the exposition of the paper. Financial support from the French Ministry of Research (Action concertée incitative) is gratefully acknowledged.

${ }^{\dagger}$ CNRS-CREST and ICER, gajdos@ensae.fr

‡EUREQua, CNRS-Université Paris I Panthéon-Sorbonne, Corresponding author: 106-112 bd de l'Hôpital, 75647 Paris Cedex 13, France, Tel: +33 (0)1 440782 04, Fax: +33 (0)1 440782 31, jmtallon@univ-paris1.fr

${ }^{\S}$ EUREQua, CNRS-Université Paris I Panthéon-Sorbonne, vergnaud@univ-paris1.fr
} 
In this paper, we adopt a new approach to modelling decision under uncertainty. We seek to explain the kind of behavior observed in Ellsberg's experiments by developing a setting in which the decision maker's preferences are defined on pairs (act, information). We thus assume that the decision maker can rank acts in different informational settings, the latter being modelled explicitly, and derive axiomatically a new decision criterion.

We now define more precisely what we call an informational state. First, we assume as usual that the sources of uncertainty can be captured by a set of states of nature. Second, we concentrate our attention on situations in which the information can be represented by a set of probability distributions over the state space, together with an anchor or reference point. To illustrate this idea, take Ellsberg's three-color urn experiment (Ellsberg (1961)), in which the decision maker is told that there are 30 red balls and 60 black or yellow balls in the urn, the draw of a roulette wheel (with number from 0 to 60 ) giving the number of black balls. Then, the set of priors that is appropriate to model the available information is simply the set of all probability distributions that place $\frac{1}{3}$ on Red. The anchor of this set is also rather naturally the distribution $\left(\frac{1}{3}, \frac{1}{3}, \frac{1}{3}\right)$. Our main modelling assumption, that the decision maker has preferences over pairs (act, information) asserts that the decision maker is able to compare say "betting on Black when the information is as above" versus "betting on Black when the composition of the urn is known to be $\left(\frac{1}{3}, \frac{1}{6}, \frac{1}{2}\right)$ ". This notion of imprecise information represented by a set of priors and an anchor is the same as the notion discussed in Hansen et alii (2001) and Wang (2003). Hansen and Sargent (2002) provide another instance in which this modelling approach is natural, reflecting "agents' fear of model misspecification":

\section{“(...) the agent regards the model [for macroeconomists, a discrete time model is a probability distribution over a sequence of vectors] as an approximation to another unknown "true" model. The agent thinks his model is a good approximation in the sense that the data are generated by another model that belongs to a vaguely specified set of models near the approximating model." \\ Hansen and Sargent (2002), Introduction to Elements of Robust Control and Filtering for Macroeconomics.}

With this setting in place, we suggest a partial order on the sets of priors with a common anchor that reflects the degree of uncertainty, or imprecision in the information, the decision maker faces. Intuitively, assuming that the information available to the decision maker can be expressed by a set of priors, set inclusion seems to be a good candidate to rank situations according to the information imprecision. However, as we will argue below, this order is too strong. As in situations of risk in which the definition of risk aversion is based on the comparison of two distributions that have a common mean, our definition of aversion towards imprecision is based on the comparison of sets of probabilities (according to set inclusion) that have the same anchor. Our axiom of aversion towards imprecision states that the decision maker prefers 
an act in a situation to the same act in a less precise situation (meaning a situation with the same anchor but with a "larger" set of priors). In our representation theorem we establish that a decision maker exhibiting aversion towards imprecision behaves as if evaluating an act by the minimum over a revealed set of probability distributions of its expected utility. The revealed set of probability distributions is a subset of the set of admissible probability distributions. An extremely pessimistic agent will keep the entire set, i.e., the revealed set of priors will be equal to the initial set of admissible priors. Conversely, a decision maker whose choices are not affected by the imprecision of the situation reduces any prior set of probability distributions as much as possible and ends up acting only on the basis of the anchor. Finally, we characterize axiomatically a more specific decision criterion that is to take a convex combination of the minimal expected utility with respect to probability distributions in the set of objectively admissible probability distributions and of the expected utility with respect to the anchor. The coefficient of the combination is a degree of pessimism.

A main contribution of the paper is therefore to provide a model in which the information available to the decision maker is explicitly taken into account in his choice behavior. This is of interest for applications of decision theory under uncertainty to problems in which data are available although not precise enough to be encapsulated in the form of a single probability distribution. Indeed, when it comes to applications, there seems to be a need to anchor prior beliefs into observed data while the usual bayesian approach does not give any information as to what prior beliefs should be formed.

Our approach allows a certain flexibility as to which revealed priors are compatible with the information: this is important as it leaves room to model different attitudes towards imprecision, and allows for decisions that are not necessarily biased towards extreme conservatism. Take for instance the global warming problem. Scientific evidence has somewhat restricted the set of possible values for important parameters, without being able, at this stage, to actually assess what are the exact effects of emission of various gas on the average temperature. Taking this evidence into account and applying the maxmin expected utility approach would then "uniquely" determine the optimal (conservative) environmental policy, leaving no room for any influence of the society attitude towards uncertainty. In our setting however, the attitude towards imprecision of the scientific evidence is an important element that, together with the evidence itself, dictates the choice of the optimal environmental policy. Different societies, with different degree of imprecision aversion, will choose different policies. More generally, we believe that modelling scientific uncertainty requires the type of approach formulated here, as it seems difficult to deal with such uncertainties in a probabilistic fashion: what does it mean for a scientific theory to be valid with probability $\frac{1}{3}$ ? In our approach on the other hand, one can associate a prior with a theory (the reference prior being the dominant theory at the time) and simply consider all theories (that have passed some minimal adequacy tests) as possible.

Another important consequence of the approach followed in the paper is that it enables one 
to perform comparative statics exercise, in which the imprecision of the information has changed. This is indeed an important advantage over a purely subjectivist approach. Many results in the literature on portfolio choice (under risk) for instance are based on some comparative statics on the riskiness of the situation or on the degree of risk aversion. An application of the results of this paper would be precisely to revisit some of this literature assuming imprecision. Interestingly, the setting developed might provide some insights into, for example, the revision of the optimal composition of a portfolio when market uncertainty increases or decreases.

Organization of the paper. The paper is organized as follows. In Section 2, we introduce our informational setup, and define a partial order on information, that reflects the accuracy or conversely the imprecision of the information, independent of the decision maker's preferences. In Section 3, we present our main results. We first develop our formal setting, which requires an operation of encoding states of nature of various situations into the set of integers. We next present our decision criterion informally (Section 3.2). In Section 3.3 we provide an axiomatic characterization of this criterion. We then discuss some properties of the revealed set of priors. Next, we develop a more specific result, providing a simple functional form for the criterion. We end the section with a study of comparative imprecision aversion. The last section contains a discussion of the relationship with the literature as well as some concluding remarks. Proofs are gathered in an appendix.

\section{Sets of priors with an anchor}

The decision problems we consider in this paper can be decomposed into two components: first the possible actions the decision maker might take and, second, the available information about the sources of uncertainty. In this section we concentrate on this second aspect and set up our general framework for representing uncertainty. Uncertainty is represented through a family of probability distributions together with a reference prior or anchor. It is a prior that belongs to the (convex hull of the) given set of priors and has a particular salience in the decision problem at hand.

\subsection{Representation of information and some examples}

Let $S$ be a set of states of nature, $\Sigma=2^{S}$ and let $\Delta(S)$ be the simplex on $S$. Let $\mathbb{P}$ be the set of closed (in the weak convergence topology) subsets of $\Delta(S)$. An element $\mathcal{P}$ of $\mathbb{P}$ is the set of all the probability distributions that are compatible with the available information in a particular decision problem. We will refer to this set as the "objective" set of priors. We consider finite settings in the sense that the number of relevant states of nature for a given problem is finite. Hence, the sets $\mathcal{P}$ we will consider have the property that their support $S(\mathcal{P})=\cup_{p \in \mathcal{P}} S u p p(p)$ are finite. Let $\mathcal{C}$ be the set of closed sets of priors with finite support. We assume that the information available about the situation at hand also allows one to identify 
an anchor or center, i.e., a probability distribution over $S$ that is (explicitly or by default) the baseline scenario. A situation will be the given of a pair $[\mathcal{P}, c]$ of a set of priors in $\mathcal{C}$, together with an anchor $c$, such that $c \in c o(\mathcal{P})$, where $c o(A)$ is the convex hull of $A$ for any set $A$. Let $\mathbb{S}$ be the set of all such possible situations.

To start with well-known examples, consider Ellsberg's two experiments, which are the usual motivation for studying models of decision under uncertainty that cannot be reduced to decision under risk.

Example 1 (Ellsberg's two color urns) A decision maker faces two urns containing a hundred balls (either black or white). He is told that there are 50 white balls and 50 black balls in the first urn, while the proportion of each color in the second urn is unknown. In both cases there are two states of nature: "the ball drawn is white" and "the ball drawn is black". The information about the known urn can be represented by the single probability measure $(.5, .5)$. A natural description of the second urn is to consider that all probability distributions over black and white balls are possible, i.e., the set of priors is the entire simplex. Based on symmetry, the distribution $(.5, .5)$ is also a natural anchor for this problem.

Example 2 (Ellsberg's three color urn) A decision maker has to bet over the color of a ball drawn from an urn that contains 30 red balls and 60 black and yellow balls in unspecified proportions. The decision maker hence knows that $\operatorname{Pr}($ Red $)=\frac{1}{3}$, and nothing else, which constitutes the set of priors compatible with the available information. As in the previous example, based on symmetry reasons, the center of this set could be taken to be the distribution $\left(\frac{1}{3}, \frac{1}{3}, \frac{1}{3}\right)$.

In the two examples above, it seems natural to appeal to symmetry considerations to determine the anchor of the set of priors. ${ }^{1}$ However, this is not necessarily the case. The following example captures the idea that agents might only have estimates of some important parameters (e.g., the law of motion of the system in a macroeconomic setting, see Hansen and Sargent (2002)).

Example 3 (Statistical inference) Consider the common practice of sampling a given population to assess the probability $p$ of appearance of a particular feature in this population. The common practice in statistics is to consider as "possible" all the parameter values that fall into the 95\% confidence interval around the estimate of $p$. Thus, the set of priors is this interval and the anchor the estimate of $p$.

Another example that can be treated in our setting is the case of two-stage lotteries. Segal (1987) in particular argued that both Allais and Ellsberg paradoxes could be explained through a relaxation of the reduction of compound lotteries axiom. When a second order probability distribution is available, our set of priors can be taken to be the support of this second order

\footnotetext{
${ }^{1}$ We refer the reader to the concluding section for a discussion of this point.
} 
distribution and the anchor to be the mean of that distribution. This interpretation is strongly related to Klibanoff, Marinacci, and Mukerji (2003)'s work, whose discussion is postponed to Section 4. Finally, consider the case in which the decision maker asks their opinion to different experts, who come up with different assessments of the situation.

Example 4 (Aggregation of expert's opinions) A decision maker asks two experts to assess the probability of occurrence of a given event. The first expert comes up with the evaluation $(1,0)$ (the event will occur with probability 1) while the second expert comes up with the evaluation $(0,1)$ (the event will occur with probability 0). The decision maker, acknowledging the disagreement among the experts will keep these two distributions as possible. The center of this set might depend on the reliability of the experts. If both are equally reliable, the center is simply the distribution $\left(\frac{1}{2}, \frac{1}{2}\right)$.

This example can be extended to models representing situations in which scientific theories compete for explaining a particular phenomenon. Scientific theories are then viewed as probability distributions over a state space. The set of priors therefore amounts to the set of theories and the anchor is then the dominant theory, challenged by the new ones.

\subsection{Comparison of imprecise information}

The representation of uncertainty through sets of priors has a direct implication for the comparison of two situations. A situation will rather naturally be considered more imprecise than an other if the set of probability distributions considered possible in the second situation is included in the set of probabilities in the first situation. However, mere set inclusion is not enough in our view. Indeed, our motivation here is to characterize a notion of imprecision with the idea that a decision maker will always prefer a given decision in the least imprecise situation. Hence, our definition of what it means for a situation to be more precise than an other has to be guided by what one would intuitively consider as the sensible choice behavior of a decision maker in more or less imprecise situations. In other words, we need to define the notion of "more imprecise than" keeping in mind the type of choice behavior we want to analyze. Thus, although it seems sensible to say that the situation in which the decision maker knows that there are 1 white and 99 black balls in an urn is more precise than the situation in which he has no information whatsoever on the proportion of white and black balls, it seems also sensible to assume that the decision maker would prefer to bet on white in the unknown urn rather than in the known urn. This gives rise to the definition of a center preserving increase in imprecision:

Definition 1 Let $\left[\mathcal{P}_{1}, c_{1}\right],\left[\mathcal{P}_{2}, c_{2}\right] \in \mathbb{S}$. The situation $\left[\mathcal{P}_{1}, c_{1}\right]$ is a (weak) center preserving increase in imprecision of the situation $\left[\mathcal{P}_{2}, c_{2}\right]$ if

1. $\mathcal{P}_{1}$ is more imprecise than $\mathcal{P}_{2}$, i.e., $\operatorname{co}\left(\mathcal{P}_{1}\right) \supseteq \operatorname{co}\left(\mathcal{P}_{2}\right)$.

2. $c_{1}=c_{2}$. 
This definition captures the intuition that an urn with 100 balls, in which it is known that there are at least 20 white and 20 black balls is more precise than an urn whose composition is unknown (under the assumption that the center is the same in the two situations.) Another intuitive feature that is embedded in our definition is slightly more subtle. Consider Ellsberg's two color urns experiment again and assume there are two balls in each urn. The situation can be described by the set $\mathcal{P}_{1}=\{(1,0),(0,1)\}$ of probability distributions over $\{$ White, Black $\}$ and center $\left(\frac{1}{2}, \frac{1}{2}\right)$. Now consider the same story, but with three balls in each urn. The set of compatible priors is $\mathcal{P}_{2}=\left\{(1,0),\left(\frac{2}{3}, \frac{1}{3}\right),\left(\frac{1}{3}, \frac{2}{3}\right),(0,1)\right\}$ and its center is $\left(\frac{1}{2}, \frac{1}{2}\right)$. Intuitively, the imprecision of the two situations is the same, as intuition suggests that the number of balls is immaterial here, and indeed our definition asserts precisely that these two situations are identical as far as imprecision is concerned, since the convex hull of the two sets coincide and their centers are identical.

Finally, we illustrate our definition with respect to sampling. Take two situations, in which an econometrician has estimated a parameter and has found the same value. In the first situation, he has estimated the parameter on a sample of say a hundred observations, while in the second he has estimated it on a sample of two hundred observations. Then, taking the point estimate as the center and the $95 \%$ confidence interval as the set of priors, it is obvious to check that the first situation is a center preserving increase in imprecision of the second situation.

\section{Representation theorems}

In this section, we first introduce our decision setting. Next, we describe the decision criterion informally and discuss some of its properties. We then turn to the axiomatic characterization of the criterion. We next discuss properties of revealed beliefs. Strengthening our axiom of aversion to imprecision leads to another representation theorem. We close the section by presenting results on comparative aversion towards imprecision.

\subsection{Setup}

Our aim is to allow choices between acts involving different state spaces. For instance, we want to model the choice between "bet 50 euros that a ball drawn randomly from an urn containing 30 red balls and 60 black or yellow balls in unknown proportion is red" which we represent as act $g$ with information $\left[\mathcal{P}_{g}, c_{g}\right]$ that is defined on the state space $S_{g}=\{$ Red,Black, Yellow $\}$, and "bet 100 euros that a ball drawn randomly from an urn containing 100 white and black balls in unknown proportion is black" which we represent as act $f$ with information $\left[\mathcal{P}_{f}, c_{f}\right]$ that is defined on the state space $S_{f}=\{$ Black, White $\}$. The formal difficulty is that the probabilistic information is not defined on the same state space. A way to proceed is to use $\mathbb{N}$ as an "encoding space", that is used to embed all the information in the same space. On our example, this simply means that we encode the different colors in different urns through integers, i.e., \{Red in urn 
I $\}$ is state $1,\{$ Black in urn I $\}$ is state $2,\{$ Yellow in urn I $\}$ is state $3,\{$ Black in urn II $\}$ is state 4 , and $\{$ White in urn II $\}$ is state 5 . We will make sure in our axiomatic construction that the specific encoding used is immaterial, in the sense that choice behavior does not depend on it.

As Gilboa and Schmeidler (1989) among others we use the framework of Anscombe and Aumann (1963). This is mostly for the sake of simplicity, as it enables us to build on their representation theorem. Let $X$ be a set (the set of outcomes) and let $Y$ be the set of distributions over $X$ with finite support (roulette lotteries). An act $f$ is a mapping from $S$ to $Y$. We denote by $\mathcal{A}$ the set of acts (horse lotteries). Let $k_{y}$ be the constant act that gives the lottery $y \in Y$ in all states, and $\mathcal{A}^{c}$ the set of constant acts. We denote $\delta_{x}$ the lottery giving $x \in X$ with probability one. Finally, for $E \subset S$, let $f_{E} g$ be the act giving $f(s)$ is $s \in E$ and $g(s)$ otherwise.

The decision maker's preferences is a binary relation $\succeq$ over $\mathcal{A} \times \mathbb{S}$, that is on couples $(f,[\mathcal{P}, c])$. As usual, $\succ$ and $\sim$ denote the asymmetric and symmetric parts, respectively, of $\succeq$.

Getting back to the example we started this section with, the bets are given by $\left(g,\left[\mathcal{P}_{g}, c_{g}\right]\right)$ and $\left(f,\left[\mathcal{P}_{f}, c_{f}\right]\right)$, where the values of $f$ and $g$ are defined as follows ( $X$ is equal to the set of real numbers):

\begin{tabular}{|c|c|c|c|c|c|c|}
\hline$s$ & 1 & 2 & 3 & 4 & 5 & $s>5$ \\
\hline$g$ & $\delta_{50}$ & $\delta_{0}$ & $\delta_{0}$ & $\delta_{0}$ & $\delta_{0}$ & $y$ \\
\hline$f$ & $\delta_{0}$ & $\delta_{0}$ & $\delta_{0}$ & $\delta_{100}$ & $\delta_{0}$ & $z$ \\
\hline
\end{tabular}

with $y, z \in Y$, and:

$$
\begin{aligned}
& {\left[\mathcal{P}_{g}, c_{g}\right]=\left[\left\{\left(\frac{1}{3}, p, \frac{2}{3}-p, 0,0,0, \ldots\right) \mid p \in\left[0, \frac{2}{3}\right]\right\},\left(\frac{1}{3}, \frac{1}{3}, \frac{1}{3}, 0,0,0, \ldots\right)\right]} \\
& {\left[\mathcal{P}_{f}, c_{f}\right]=\left[\{(0,0,0, p, 1-p, 0, \ldots) \mid p \in[0,1]\},\left(0,0,0, \frac{1}{2}, \frac{1}{2}, 0, \ldots\right)\right]}
\end{aligned}
$$

Observe that $f$ and $g$ have been arbitrarily defined for $s>5$, since we need to define acts on the entire state space $S$. However, our axioms will ensure that their definition outside the support of the information set is irrelevant (this is a consequence of Axiom 2, below). Then, $\left(g,\left[\mathcal{P}_{g}, c_{g}\right]\right) \succeq\left(f,\left[\mathcal{P}_{f}, c_{f}\right]\right)$ means that act $g$ in the situation $\left[\mathcal{P}_{g}, c_{g}\right]$ is preferred to act $f$ in situation $\left[\mathcal{P}_{f}, c_{f}\right]$.

\subsection{Decision criterion}

The decision criterion that we will characterize axiomatically in the next section states that $\left(f,\left[\mathcal{P}_{i}, c_{i}\right]\right) \succeq\left(g,\left[\mathcal{P}_{j}, c_{j}\right]\right)$ if, and only if:

$$
\min _{p \in \mathcal{F}_{\left[\mathcal{P}_{i}, c_{i}\right]}} \int u \circ f d p \geq \min _{p \in \mathcal{F}_{\left[\mathcal{P}_{j}, c_{j}\right]}} \int u \circ g d p .
$$

In this expression, $u$ is the utility function and $\mathcal{F}_{\left[\mathcal{P}_{i}, c_{i}\right]}$ is a subfamily of $\mathcal{P}_{i}$ that can be interpreted as the subjective family of priors revealed by the decision maker through choice behavior. The decision criterion implies that the decision maker acts as if he were using the maxmin expected utility model but with respect to a family of priors that is a subset of the given set of priors, representing available information. 
At one extreme, the decision maker's revealed set of priors reduces to a singleton. In this case, the structure imposed on the model requires that this prior is the anchor of the objective set of priors. The criterion then reduces to the expected utility criterion: the decision maker evaluates an act in a given situation by taking the expected utility of that act with respect to the anchor. This behavior corresponds to neutrality towards imprecision of the prior information. In particular, such a decision maker would be indifferent between an act $f$ in a situation $[\mathcal{P}, c]$ and the same act in the situation $\left[\mathcal{P}^{\prime}, c^{\prime}\right]$ which is a center preserving increase in imprecision of $[\mathcal{P}, c]$. At the other extreme, the decision maker's revealed set of priors is the same as the one he was given to begin with, i.e., $\mathcal{F}_{[\mathcal{P}, c]}$ is equal to $\mathcal{P}$. The criterion is then the maxmin expected utility criterion on the entire family of priors representing the situation, axiomatized in a context similar to ours in Wang (2003). Such a decision maker could be said to have an extreme aversion towards imprecision. Our model is hence flexible enough to accommodate bayesian (expected utility) behavior on the one hand and extreme pessimism on the other hand. In between these two extremes lie all the possible attitudes towards imprecision: the extent to which the decision maker "shrinks" the given family can be seen as a measure of his aversion towards imprecision.

The criterion we obtain looks similar to Gilboa and Schmeidler (1989) multiple prior model. However, the setting and the axiomatic characterization are rather different (in particular, their axiom of uncertainty aversion is based on mixture of acts.) We postpone the discussion of the relationship between the two approaches after the presentation of the axiomatic characterization of our criterion.

\subsection{Axiomatic characterization}

In this section, we develop the axiomatic characterization of the criterion introduced above. Our axioms bear on couples (act, situation) which is unusual in the literature (although see Wang (2003)), but are otherwise interpretable in much the same way as in the more traditional von Neumann-Morgenstern setting. We begin by assuming that the preference relation is a weak order.

Axiom 1 (Weak order) $\succeq$ is complete and transitive.

The second axiom states that the way the encoding of the states of nature into $\mathbb{N}$ is done does not matter and requires some further definitions. The aim of this construction is to ensure that the only relevant information upon which the decision maker is acting is the set of induced probability distributions over outcomes and not the states of the world themselves.

For any $\varphi$ onto mapping from $S$ to $S$ (i.e $\varphi(S)=S$ ), for any $f \in \mathcal{A}$, we say that $f$ is $\varphi$-measurable if $f(s)=f\left(s^{\prime}\right)$ for all $s, s^{\prime} \in S$ such that $\varphi(s)=\varphi\left(s^{\prime}\right)$. We will use the following notation.

\section{Notation 1}


- For all $f \in \mathcal{A}$, and all $\varphi$ onto mapping from $S$ to $S$ such that $f$ is $\varphi$-measurable, $f^{\varphi}$ is defined by $f^{\varphi}(s)=f\left(s^{\prime}\right)$ where $s^{\prime} \in \varphi^{-1}(s)$ for all $s \in S$.

- For any $p \in \Delta(S)$ and $[\mathcal{P}, c] \in \mathbb{S}$ and $\varphi$ onto mapping from $S$ to $S, p^{\varphi}$ is defined by $p^{\varphi}(s)=p\left(\varphi^{-1}(s)\right)$ for all $s \in S$ and $\mathcal{P}^{\varphi}$ by $\mathcal{P}^{\varphi}=\left\{q \in \Delta(S) \mid q=p^{\varphi}, p \in \mathcal{P}\right\}$. Furthermore, we will note $[\mathcal{P}, c]^{\varphi}=\left[\mathcal{P}^{\varphi}, c^{\varphi}\right]$, and $(f,[\mathcal{P}, c])^{\varphi}=\left(f^{\varphi},[\mathcal{P}, c]^{\varphi}\right)$.

If $\varphi$ is a bijection, note that for all $p \in \Delta(S)$, there is a unique $q \in \Delta(S)$ such that $q^{\varphi}=p$.

Axiom 2 (Equivalence indifference) For all $f, g \in \mathcal{A},[\mathcal{P}, c] \in \mathbb{S}$,

- $(f,[\mathcal{P}, c]) \sim\left(f_{S(\mathcal{P})} g,[\mathcal{P}, c]\right)$

- if $\varphi$ is an onto mapping from $S$ to $S$ such that $f$ is $\varphi$-measurable, then $(f,[\mathcal{P}, c]) \sim$ $(f,[\mathcal{P}, c])^{\varphi}$.

Axiom 2 implies in particular that for all constant act $f \in \mathcal{A}^{c}$, for all $\left[\mathcal{P}_{1}, c_{1}\right],\left[\mathcal{P}_{2}, c_{2}\right] \in \mathbb{S}$, $\left(f,\left[\mathcal{P}_{1}, c_{1}\right]\right) \sim\left(f,\left[\mathcal{P}_{2}, c_{2}\right]\right)$.

Example 5 Consider Ellsberg's two urns example and encode $\{$ Black in urn I\} by 1, \{White in urn I\} by 2, \{Black in urn II\} by 3, \{White in urn II \} by 4. The preference of the decision maker to bet on, say, Black in the known urn rather than on Black in the urn with an unknown proportion of black and white balls would be written: $\left(f,\left[\mathcal{P}_{1}, c_{1}\right]\right) \succeq\left(g,\left[\mathcal{P}_{2}, c_{2}\right]\right)$ where $f$ is the act defined by $f(1)=\delta_{1}$ and $f(s)=\delta_{0}$ for all $s \neq 1, g$ is the act defined by $g(3)=\delta_{1}$ and $g(s)=\delta_{0}$ for all $s \neq 3, \mathcal{P}_{1}=\left\{\left(\frac{1}{2}, \frac{1}{2}, 0,0,0, \ldots\right)\right\}=\left\{c_{1}\right\}, \mathcal{P}_{2}=\{(0,0, p, 1-p, 0, \ldots) \mid p \in[0,1]\}$ and $c_{2}=\left(0,0, \frac{1}{2}, \frac{1}{2}, 0, \ldots\right)$.

An equivalent description for the comparison at hand would be to encode $\{$ White in urn I or II\} by 1, \{Black in urn I\} by 2, and \{Black in urn II $\}$ by 3. Let $f^{\prime}$ be defined by $f^{\prime}(2)=\delta_{1}$ and $f^{\prime}(s)=\delta_{0}$ for all $s \neq 1$, and let $g^{\prime}$ be defined by $g^{\prime}(3)=\delta_{1}$ and $g^{\prime}(s)=\delta_{0}$ for all $s \neq 3$. Let $\mathcal{P}_{1}^{\prime}=$ $\left\{\left(\frac{1}{2}, \frac{1}{2}, 0,0, \ldots\right)\right\}, \mathcal{P}_{2}^{\prime}=\{(p, 0,1-p, 0, \ldots) \mid p \in[0,1]\}, c_{1}^{\prime}=\left(\frac{1}{2}, \frac{1}{2}, 0,0, \ldots\right)$ and $c_{2}^{\prime}=\left(\frac{1}{2}, 0, \frac{1}{2}, 0, \ldots\right)$. Define $\varphi$ by $\varphi(1)=2, \varphi(2)=\varphi(4)=1, \varphi(3)=3$, and $\varphi(s)=s-1$ for all $s \geq 4$. Then, observing that $f^{\prime}=f^{\varphi}, g^{\prime}=g^{\varphi}$ and $\left[\mathcal{P}_{i}^{\prime}, c_{i}^{\prime}\right]=[\mathcal{P}, c]^{\varphi_{i}}$, Axiom 2 imposes that

$$
\left(f,\left[\mathcal{P}_{1}, c_{1}\right]\right) \succeq\left(g,\left[\mathcal{P}_{2}, c_{2}\right]\right) \Leftrightarrow\left(f^{\prime},\left[\mathcal{P}_{1}^{\prime}, c_{1}^{\prime}\right]\right) \succeq\left(g^{\prime},\left[\mathcal{P}_{2}^{\prime}, c_{2}^{\prime}\right]\right)
$$

The next axiom is an independence axiom that requires to define the notion of probabilistic mixture of situations. We first introduce some notation.

Notation 2 Let $\mathcal{P}, \mathcal{P}^{*} \in \mathbb{P}$. For all $p \in \mathcal{P}$ and $p^{*} \in \mathcal{P}^{*}$, we denote by $\alpha p+(1-\alpha) p^{*}$ the probability distribution with support $S(\mathcal{P}) \cup S\left(\mathcal{P}^{*}\right)$, defined by:

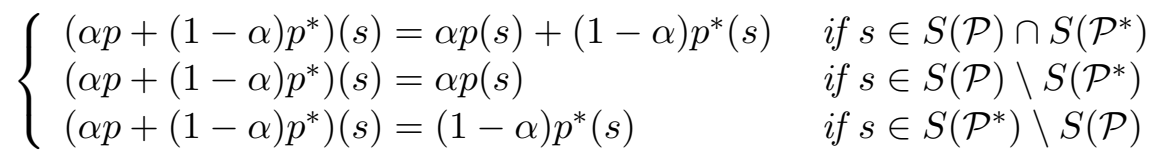


Furthermore, we denote by $\alpha \mathcal{P}+(1-\alpha) \mathcal{P}^{*}$ the set:

$$
\left\{q \in \Delta\left(S\left(\mathcal{P} \cup \mathcal{P}^{*}\right)\right) \mid q=\alpha p+(1-\alpha) p^{*}, p \in \mathcal{P}, p^{*} \in \mathcal{P}^{*}\right\}
$$

Definition 2 (Probability mixture) Let $[\mathcal{P}, c],\left[\mathcal{P}^{*}, c^{*}\right] \in \mathbb{S}$, and $\alpha \in[0,1]$. The $\alpha:(1-\alpha)$ mixture of $[\mathcal{P}, c]$ and $\left[\mathcal{P}^{*}, c^{*}\right]$, denoted $\alpha[\mathcal{P}, c]+(1-\alpha)\left[\mathcal{P}^{*}, c^{*}\right]$, is defined by :

$$
\alpha[\mathcal{P}, c]+(1-\alpha)\left[\mathcal{P}^{*}, c^{*}\right]=\left[\alpha \mathcal{P}+(1-\alpha) \mathcal{P}^{*}, \alpha c+(1-\alpha) c^{*}\right]
$$

Example 6 Consider Ellsberg's two-urn experiment again, using the first encoding described in Example 5. The $\alpha:(1-\alpha)$-mixture of $\left[\mathcal{P}_{1}, c_{1}\right]$ and $\left[\mathcal{P}_{2}, c_{2}\right]$ is the situation given by

$$
\alpha \mathcal{P}_{1}+(1-\alpha) \mathcal{P}_{2}=\left\{\left(\frac{1}{2} \alpha, \frac{1}{2} \alpha,(1-\alpha) p,(1-\alpha)(1-p), 0, \ldots\right) \mid p \in[0,1]\right\}
$$

and

$$
\alpha c_{1}+(1-\alpha) c_{2}=\left(\frac{1}{2} \alpha, \frac{1}{2} \alpha, \frac{1}{2}(1-\alpha), \frac{1}{2}(1-\alpha), 0, \ldots\right)
$$

Notation 3 A particular instance of a mixture, that will be heavily used in the sequel, is the $\alpha:(1-\alpha)$-mixture of $[\mathcal{P}, c]$ with $[\mathcal{P}, c]^{\varphi}$. We will denote it $[\mathcal{P}, c]_{M}^{(\alpha, \varphi)}$.

We are now in a position to state our mixture independence axiom.

Axiom 3 (Mixture independence) For all $\left[\mathcal{P}_{i}, c_{i}\right] \in \mathbb{S}, i=1,2,3$, such that $\left(S\left(\mathcal{P}_{1}\right) \cup S\left(\mathcal{P}_{2}\right)\right) \cap$ $S\left(\mathcal{P}_{3}\right)=\emptyset$, for all $\alpha \in[0,1)$, and for all $f, g \in \mathcal{A}$ such that $f(s)=g(s)$ for all $s \in S\left(\mathcal{P}_{3}\right)$,

$$
\left(f,\left[\mathcal{P}_{1}, c_{1}\right]\right) \succeq\left(g,\left[\mathcal{P}_{2}, c_{2}\right]\right) \Leftrightarrow\left(f, \alpha\left[\mathcal{P}_{1}, c_{1}\right]+(1-\alpha)\left[\mathcal{P}_{3}, c_{3}\right]\right) \succeq\left(g, \alpha\left[\mathcal{P}_{2}, c_{2}\right]+(1-\alpha)\left[\mathcal{P}_{3}, c_{3}\right]\right)
$$

When sets of priors are reduced to singletons this is the usual independence axiom. We next need another operation on sets of priors, that we dub replication.

Definition 3 (Replication) Let $[\mathcal{P}, c] \in \mathbb{S}, \alpha \in[0,1]$, and $S^{\prime} \subset \mathbb{N}$ such that $S^{\prime} \cap S(\mathcal{P})=\emptyset$. Let $\varphi$ be a bijection from $S$ to $S$ such that $\varphi(S(\mathcal{P}))=S^{\prime}$. The $(\alpha, \varphi)$-replication of $[\mathcal{P}, c]$ denoted $[\mathcal{P}, c]_{R}^{(\alpha, \varphi)}$ is defined by:

$$
[\mathcal{P}, c]_{R}^{(\alpha, \varphi)}=\left[\left\{q \in \Delta\left(S(\mathcal{P}) \cup S^{\prime}\right) \mid q=\alpha p+(1-\alpha) p^{\varphi}, p \in \mathcal{P}\right\}, \alpha c+(1-\alpha) c^{\varphi}\right]
$$

Note that $[\mathcal{P}, c]_{R}^{(1, \varphi)}=[\mathcal{P}, c]$ and $[\mathcal{P}, c]_{R}^{(0, \varphi)}=[\mathcal{P}, c]^{\varphi}$.

Example 7 Consider an urn with one ball, that could be black or white. Consider the "replication" of this urn, with one ball that could be red or green. Replication could be thought of as, for instance, taking the initial urn, duplicate it, and painting the ball red if it was originally black or green if it was originally white. Let us encode the different colors in the different urns as follows: $\{$ Black in urn I\} is state 1, \{White in urn I\} is state 2, \{Red in urn II\} is state 3, and 
$\{$ Green in urn II\} is state 4. Define $\varphi(1)=3, \varphi(2)=4, \varphi(3)=1, \varphi(4)=2$ and $\varphi(s)=s$ for all $s>4$. Then the $\frac{1}{2}$-replication is the the set $\left\{\left(\frac{1}{2}, 0, \frac{1}{2}, 0,0, \ldots\right),\left(0, \frac{1}{2}, 0, \frac{1}{2}, 0, \ldots\right\}\right.$. It corresponds to the description of the urn that is composed by putting together the two urns described. Since by definition they have the "same" composition, the only possible states are those in which either the balls are Black and Red, or the balls are White and Green, and in each case there is exactly one ball of each color.

More generally, the $\alpha$-replication of $\mathcal{P}$ would correspond (when $\alpha$ is rational) to the description of the urn composed by putting together $n$ identical urns $\{B, W\}$ and $n^{\prime}$ (identical) urns $\{R, G\}$ generated as described above, with $\alpha=\frac{n}{n+n^{\prime}}$.

Define the mixture of two acts $\alpha f+(1-\alpha) g$ as usual, i.e., it is the act giving $f(s)$ with probability $\alpha$ and $g(s)$ with probability $(1-\alpha)$ in state $s$.

Axiom 4 (Replication indifference) For all $[\mathcal{P}, c] \in \mathbb{S}$, for all replication $[\mathcal{P}, c]_{R}^{(\alpha, \varphi)}$, for all $f, g \in \mathcal{A}$ such that $f(s)=g(s)$ for all $s \in S \backslash S(\mathcal{P})$,

$$
(\alpha f+(1-\alpha) g,[\mathcal{P}, c]) \sim\left(f_{S(\mathcal{P})} g^{\varphi},[\mathcal{P}, c]_{R}^{(\alpha, \varphi)}\right)
$$

In the previous axiom, observe that $f_{S(\mathcal{P})} g^{\varphi}(s)=f(s)=g(s)$ for all $s \in S \backslash(S(\mathcal{P}) \cup \varphi(S(\mathcal{P}))$. The next axiom is a continuity axiom.

Axiom 5 (Continuity) For all $f, g, h \in \mathcal{A}$, and all $[\mathcal{P}, c] \in \mathbb{S}$, if $(f,[\mathcal{P}, c]) \succ(g,[\mathcal{P}, c]) \succ$ $(h,[\mathcal{P}, c])$, then there exist $\alpha$ and $\beta$ in $] 0,1[$ such that :

$$
(\alpha f+(1-\alpha) h,[\mathcal{P}, c]) \succ(g,[\mathcal{P}, c]) \succ(\beta f+(1-\beta) h,[\mathcal{P}, c]) .
$$

Note that $\succeq$ induces a preference relation $\succeq_{[\mathcal{P}, c]}^{\ell}$ on $Y$ which is simply the restriction of $\succeq$ on $\mathcal{A}^{c} \times\{[\mathcal{P}, c]\}$. The next axiom states that the order $\succeq$ should be monotonic when comparing couples $(f,[\mathcal{P}, c])$ and $(g,[\mathcal{P}, c])$ in which the act-component are ranked according to the $\succeq_{[\mathcal{P}, c]}^{\ell}$ order.

Axiom 6 (Monotonicity) For all $f, g \in \mathcal{A}$, and all $[\mathcal{P}, c] \in \mathbb{S}$, if $f(s) \succeq_{[\mathcal{P}, c]}^{\ell} g(s)$ for all $s \in S(\mathcal{P})$, then $(f,[\mathcal{P}, c]) \succeq(g,[\mathcal{P}, c])$.

The next axiom is rather weak and simply requires that for any situation, there exists a pair of acts that are not indifferent.

Axiom 7 (Non-degeneracy) For all $[\mathcal{P}, c] \in \mathbb{S}$, there exist $f, g \in \mathcal{A}$ such that $(f,[\mathcal{P}, c]) \succ$ $(g,[\mathcal{P}, c])$.

The axiom of aversion towards imprecision is at the heart of our construction. It relates behavior in two different situations, one being a center preserving increase in imprecision of the other, and states that the decision maker will always prefer to act in the second situation. 
Axiom 8 (Aversion towards imprecision) For all $f \in \mathcal{A},\left[\mathcal{P}_{1}, c_{1}\right],\left[\mathcal{P}_{2}, c_{2}\right] \in \mathbb{S}$ such that $\left[\mathcal{P}_{1}, c_{1}\right]$ is a center preserving increase in imprecision of $\left[\mathcal{P}_{2}, c_{2}\right],\left(f,\left[\mathcal{P}_{2}, c_{2}\right]\right) \succeq\left(f,\left[\mathcal{P}_{1}, c_{1}\right]\right)$.

Aversion towards imprecision only compares situations with the same anchor (i.e., $c_{1}=c_{2}$ ). It thus has no bite for situations which are ranked according to the set inclusion order, if they do not have the same anchor. This feature is important in understanding why the axiom allows for a whole range of attitudes towards imprecision. Our last axiom is a Pareto principle that states that if $f$ is preferred to $g$ for all singleton sets of priors included in $\mathcal{P}$, then $f$ is preferred to $g$ when information is the union of all these singletons.

Axiom 9 (Pareto) For all $[\mathcal{P}, c] \in \mathbb{S}$, if for all $p \in \mathcal{P}$, we have $(f,[\{p\}, p]) \succeq(g,[\{p\}, p])$, then $(f,[\mathcal{P}, c]) \succeq(g,[\mathcal{P}, c])$.

The next theorem is our main result. It provides a characterization of our set of axioms, in which the notion of uncertainty aversion is captured by aversion towards information imprecision.

Theorem 1 Axioms 1 to 9 hold if, and only if, there exists an unique (up to a positive linear transformation) affine function $u: Y \rightarrow \mathbb{R}$, and for all $\left[\mathcal{P}_{i}, c_{i}\right] \in \mathbb{S}$, there exist unique, non-empty, closed and convex sets $\mathcal{F}_{\left[\mathcal{P}_{i}, c_{i}\right]}$ of probability measures on $2^{S}$, satisfying

1. $\mathcal{F}_{\left[\mathcal{P}_{i}, c_{i}\right]} \subseteq \mathcal{P}_{i}$

2. For all $\varphi$ onto mapping from $S$ to $S, \mathcal{F}_{\left[\mathcal{P}_{i}, c_{i}\right]^{\varphi}}=\left(\mathcal{F}_{\left[\mathcal{P}_{i}, c_{i}\right]}\right)^{\varphi}$

3. If $\left[\mathcal{P}_{i}, c_{i}\right],\left[\mathcal{P}_{j}, c_{j}\right] \in \mathbb{S}$ are such that $S\left(\mathcal{P}_{i}\right) \cap S\left(\mathcal{P}_{j}\right)=\emptyset$, then for all $\alpha \in[0,1]$,

$$
\mathcal{F}_{\alpha\left[\mathcal{P}_{i}, c_{i}\right]+(1-\alpha)\left[\mathcal{P}_{j}, c_{j}\right]}=\alpha \mathcal{F}_{\left[\mathcal{P}_{i}, c_{i}\right]}+(1-\alpha) \mathcal{F}_{\left[\mathcal{P}_{j}, c_{j}\right]}
$$

4. If $\left[\mathcal{P}_{i}, c_{i}\right]$ is a center preserving increase in imprecision of $\left[\mathcal{P}_{j}, c_{j}\right]$ then $\mathcal{F}_{\left[\mathcal{P}_{i}, c_{i}\right]} \supseteq \mathcal{F}_{\left[\mathcal{P}_{j}, c_{j}\right]}$

5. For $[\mathcal{P}, c] \in \mathbb{S}$, for all replication $[\mathcal{P}, c]_{R}^{(\alpha, \varphi)}$,

$$
\mathcal{F}_{[\mathcal{P}, c]_{R}^{(\alpha, \varphi)}}=\left\{\alpha p+(1-\alpha) p^{\varphi} \mid p \in \mathcal{F}_{[\mathcal{P}, c]}\right\}
$$

such that for all $f, g \in \mathcal{A},\left(f,\left[\mathcal{P}_{i}, c_{i}\right]\right) \succeq\left(g,\left[\mathcal{P}_{j}, c_{j}\right]\right)$ if, and only if:

$$
\min _{\left.p \in \mathcal{F}_{\left[\mathcal{P}_{i}, c_{i}\right]}\right]} \int u \circ f d p \geq \min _{\left.p \in \mathcal{F}_{\left[\mathcal{P}_{j}, c_{j}\right]}\right]} \int u \circ g d p .
$$

The theorem provides an axiomatization of the maxmin multiple prior model where the revealed set of priors is constrained by the available information. These constraints are reflected in conditions 1 to 5 that put structure on the revealed set and will be discussed in the next section. The theorem is proved in the appendix and we simply sketch the strategy of the proof here. The 
latter consists in using Gilboa and Schmeidler (1989) result. More specifically, restricting choice among acts in a given situation and imposing Gilboa and Schmeidler's certainty independence and uncertainty aversion, we can use their result to establish that the decision criterion is of the maxmin expected utility type - Axiom 2 implying that the utility function is independent of the situation (Theorem 4 in the Appendix). We then show that our set of axioms implies Gilboa and Schmeidler's and derive the conditions on the revealed set of priors from the additional structure of our model, namely the way the decision maker processes information.

\subsection{Properties of the revealed set of priors}

Theorem 1 provides an axiomatization of the maxmin multiple prior model where the revealed set of priors is constrained by the available information. Condition 1 expresses the "natural" restriction that the revealed family be included in the objective data, which therefore provide bounds on the beliefs. As mentioned above, any revealed family from a single prior (the center) to the entire objective family is admissible.

Condition 2 is a consistency requirement that relates the revealed family in a situation $\left[\mathcal{P}_{i}, c_{i}\right]$ to the revealed family in a situation that is linked to the previous one. When $\varphi$ is a bijection, this means for instance that the revealed family is invariant to a permutation of the states if the available information is the same. To illustrate this, consider Ellsberg's three color urn example, with the following encoding of the states: $\{1\}$ is associated to Red, $\{2\}$ to Black and $\{3\}$ to Yellow. The information the decision maker has is hence $\mathcal{P}=\left\{p \in \Delta(S) \mid p(1)=\frac{1}{3}, p(2)+p(3)=\right.$ $\left.\frac{2}{3}\right\}$. Assume the center is $\left(\frac{1}{2}, \frac{1}{3}, \frac{1}{3}, 0, \ldots\right)$. Let $\varphi: S \rightarrow S$ be defined by $\varphi(1)=1, \varphi(2)=3$, $\varphi(3)=2$, and $\varphi(s)=s$ for all $s \neq 1,2,3$. We then have $[\mathcal{P}, c]^{\varphi}=[\mathcal{P}, c]$.

Condition 2 in Theorem 1 implies $\mathcal{F}_{[\mathcal{P}, c]^{\varphi}}=\left(\mathcal{F}_{[\mathcal{P}, c]}\right)^{\varphi}$ and hence we have in this particular case that $\mathcal{F}_{[\mathcal{P}, c]}=\left(\mathcal{F}_{[\mathcal{P}, c]}\right)^{\varphi}$. In our example, this simply means that for every $q \in \mathcal{F}_{[\mathcal{P}, c]^{\varphi}}$, there exists $p \in \mathcal{F}_{[\mathcal{P}, c]}$ such that $q(1)=p(1), q(2)=p(3)$ and $q(3)=p(2)$, and conversely. Hence, the revealed set of beliefs has to be "symmetric" around its center. For instance, one can have $\mathcal{F}_{[\mathcal{P}, c]}=\left\{\left(\frac{1}{3}, \frac{1}{3}-\pi, \frac{1}{3}+\pi, 0, \ldots\right) \mid \pi \in\left[-\frac{1}{6}, \frac{1}{6}\right]\right\}$, but it is not possible to have $\mathcal{F}_{[\mathcal{P}, c]}=\left\{\left(\frac{1}{3}, \frac{1}{3}-\right.\right.$ $\left.\left.\pi, \frac{1}{3}+\pi, 0, \ldots\right) \mid \pi \in\left[-\frac{1}{4}, \frac{1}{6}\right]\right\}$ since if it were the case, one would have $\left(\frac{1}{3}, \frac{7}{12}, \frac{1}{12}, 0, \ldots\right) \in \mathcal{F}_{[\mathcal{P}, c]}$ but $\left(\frac{1}{3}, \frac{7}{12}, \frac{1}{12}, 0, \ldots\right) \notin\left(\mathcal{F}_{[\mathcal{P}, c]}\right)^{\varphi}$.

Condition 3 relates the revealed family of the mixture of two situations to the two revealed families associated to these two situations and states that the mixture operation does not reduce imprecision.

Condition 4 states that, given two situations that can be compared according to the partial order defined in Section 2.2, it must be the case that the revealed set of priors in the less imprecise situation is included in the revealed set of priors in the more imprecise situation.

To illustrate Condition 5, we pursue on Ellsberg's three color urn developed in the discussion of Condition 2. Assume as there that the revealed set of beliefs is $\mathcal{F}_{[\mathcal{P}, c]}=\left\{\left(\frac{1}{3}, \frac{1}{3}-\pi, \frac{1}{3}+\right.\right.$ $\left.\pi, 0, \ldots) \mid \pi \in\left[-\frac{1}{6}, \frac{1}{6}\right]\right\}$. Consider the $\frac{1}{2}$-replication of $[\mathcal{P}, c]$, in which states $1,2,3$ are sent to 
states $3,4,5$ and vice versa. Then, Condition 5 asserts that the revealed set of priors has to be

$$
\mathcal{F}_{[\mathcal{P}, c]_{R}^{\left(\frac{1}{2}, \varphi\right)}}=\left\{\left(\frac{1}{6}, \frac{1}{6}-\frac{\pi}{2}, \frac{1}{6}+\frac{\pi}{2}, \frac{1}{6}, \frac{1}{6}-\frac{\pi}{2}, \frac{1}{6}+\frac{\pi}{2}, 0, \ldots\right) \mid \pi \in\left[-\frac{1}{6}, \frac{1}{6}\right]\right\}
$$

This is to be compared with the revealed family when that same situation $[\mathcal{P}, c]$ is mixed (with weight $\frac{1}{2}$ ) with $[\mathcal{P}, c]^{\varphi}$, as given by Conditions 2 and 3 :

$$
\mathcal{F}_{[\mathcal{P}, c]_{M}^{\left(\frac{1}{2}, \varphi\right)}}=\left\{\left(\frac{1}{6}, \frac{1}{6}-\frac{\pi}{2}, \frac{1}{6}+\frac{\pi}{2}, \frac{1}{6}, \frac{1}{6}-\frac{\pi^{\prime}}{2}, \frac{1}{6}+\frac{\pi^{\prime}}{2}, 0, \ldots\right) \mid \pi \in\left[-\frac{1}{6}, \frac{1}{6}\right], \pi^{\prime} \in\left[\frac{1}{6}, \frac{1}{6}\right]\right\}
$$

The fact that $\mathcal{F}_{[\mathcal{P}, c]_{M}\left(\frac{1}{2}, \varphi\right)} \subset \mathcal{F}_{[\mathcal{P}, c]_{R}}\left(\frac{1}{2}, \varphi\right)$ is a direct consequence of Lemma 1 in the Appendix, which states that the mixture operation yields situations that are center preserving increases in imprecision compared to the replication operation.

\subsection{Characterizing aversion towards imprecision}

In this section, we strengthen the axiom of aversion to imprecision of information and obtain a more specific representation theorem.

Axiom 10 (Dominance) For all $f, g \in \mathcal{A},\left[\mathcal{P}_{1}, c_{1}\right],\left[\mathcal{P}_{2}, c_{2}\right] \in \mathbb{S}$, if $\left(f,\left[\left\{c_{1}\right\}, c_{1}\right]\right) \succeq\left(g,\left[\left\{c_{2}\right\}, c_{2}\right]\right)$ and for all $p \in \mathcal{P}_{1}$, there exists $q \in \mathcal{P}_{2}$ such that $(f,[\{p\}, p]) \succeq(g,[\{q\}, q])$, then $\left(f,\left[\mathcal{P}_{1}, c_{1}\right]\right) \succeq$ $\left(g,\left[\mathcal{P}_{2}, c_{2}\right]\right)$.

It is easy to show that this axiom is indeed a strengthening of the aversion to imprecise information axiom and actually that it implies Axioms 8 and 9.

Theorem 2 Axioms 1 to 7 , and 10 hold if, and only if, there exist an unique (up to a positive linear transformation) affine function $u: Y \rightarrow \mathbb{R}$, and $\alpha \in[0,1]$, such that for all $\left[\mathcal{P}_{1}, c_{1}\right],\left[\mathcal{P}_{2}, c_{2}\right] \in \mathbb{S}$, for all $f, g \in \mathcal{A}$,

$$
\left(f,\left[\mathcal{P}_{1}, c_{1}\right]\right) \succeq\left(g,\left[\mathcal{P}_{2}, c_{2}\right]\right)
$$

if, and only if,

$$
\alpha \min _{p \in \operatorname{co}\left(\mathcal{P}_{1}\right)} \int u \circ f d p+(1-\alpha) \int u \circ f d c_{1} \geq \alpha \min _{p \in \operatorname{co}\left(\mathcal{P}_{2}\right)} \int u \circ g d p+(1-\alpha) \int u \circ g d c_{2}
$$

The parameter $\alpha$ in the above representation theorem can be interpreted as a degree of pessimism of the decision maker. If $\alpha=0$, he behaves as an expected utility maximizer with respect to the anchor probability, while if $\alpha=1$, he behaves as a maximizer of the minimum expected utility with respect to all the distributions compatible with his information. 


\subsection{Comparative imprecision aversion}

In this section, we show that a decision maker is more averse towards imprecision than another if, in any given situation, his revealed set of priors is included in the second decision maker's revealed set. Assume from now on that the set of consequences $X$ is equal to $[0, M] \subset \mathbb{R}$ and that preferences respect the natural order on $[0, M]$, i.e., if $x, y \in[0, M]$ and $x>y$, then, in any possible situation, the decision maker prefers getting the constant degenerate lottery $k_{\delta_{x}}$ giving him $x$ in all the states to getting $k_{\delta_{y}}$.

Let $Y_{B}$ be the set of lotteries over $\{0, M\}$, i.e., the set of lotteries whose outcomes consist only of the two extreme prizes. $y \in Y_{B}$ can be written $(0, p ; M, 1-p)$. Similarly, let $\mathcal{A}_{B}$ be the set of acts defined on $Y_{B}$, i.e., acts that can be written $f(s)=y_{s}$ with $y_{s}=\left(0,1-p_{s} ; M, p_{s}\right) \in Y_{B}$ for all $s \in S$.

Definition 4 Let $\succeq_{a}$ and $\succeq_{b}$ be two preference relations defined on $\mathcal{A} \times \mathbb{S}$. We say that $\succeq_{b}$ is more imprecision averse than $\succeq_{a}$ if for all $y \in Y_{B}$ and all $f \in \mathcal{A}_{B}$, and for all $[\mathcal{P}, c] \in \mathbb{S}$,

$$
\left(k_{y},[\mathcal{P}, c]\right) \succeq_{a}(f,[\mathcal{P}, c]) \Rightarrow\left(k_{y},[\mathcal{P}, c]\right) \succeq_{b}(f,[\mathcal{P}, c])
$$

and

$$
\left(k_{y},[\mathcal{P}, c]\right) \succ_{a}(f,[\mathcal{P}, c]) \Rightarrow\left(k_{y},[\mathcal{P}, c]\right) \succ_{b}(f,[\mathcal{P}, c])
$$

where $k_{y}$ is the constant act giving the binary lottery $y$ in all states.

This definition differs from the one in Ghirardato and Marinacci (2002) and Epstein (1999), in that we restrict attention to comparisons among binary lotteries and binary acts.

Definition 5 Let $(f,[\mathcal{P}, c]) \in \mathcal{A} \times \mathbb{S}$. Call the probabilistic binary equivalent of $(f,[\mathcal{P}, c])$ the lottery $\operatorname{Pe}(f,[\mathcal{P}, c])=(0,1-p ; M, p) \in Y_{B}$ such that $(f,[\mathcal{P}, c]) \sim\left(k_{P e(f,[\mathcal{P}, c])},[\mathcal{P}, c]\right)$, where $k_{P e(f,[\mathcal{P}, c])}$ is the constant act giving $\operatorname{Pe}(f,[\mathcal{P}, c])$.

Under continuity, such a probabilistic binary equivalent always exists. Probabilistic binary equivalent can be associated to the probability $p$ of getting $M$ in the lottery $\operatorname{Pe}(f,[\mathcal{P}, c])$. Denote $\Pi(f,[\mathcal{P}, c])$ this quantity, i.e., $\operatorname{Pe}(f,[\mathcal{P}, c])=(0,1-\Pi(f,[\mathcal{P}, c]) ; M, \Pi(f,[\mathcal{P}, c]))$. Normalize utilities so that $u\left(\delta_{0}\right)=0$ and $u\left(\delta_{M}\right)=1$. It is straightforward to see that for $\succeq$ satisfying all the axioms of Theorem 1 , if $\left[\mathcal{P}_{1}, c_{1}\right]$ is a center preserving imprecision increase of $\left[\mathcal{P}_{2}, c_{2}\right]$, then, for all $f \in \mathcal{A}, \Pi\left(f,\left[\mathcal{P}_{2}, c_{2}\right]\right) \leq \Pi\left(f,\left[\mathcal{P}_{1}, c_{1}\right]\right)$.

Theorem 3 Let $\succeq_{a}$ and $\succeq_{b}$ be two preference relations defined on $\mathcal{A} \times \mathbb{S}$, satisfying all axioms of Theorem 1. Then, the following assertions are equivalent

(i) $\succeq_{b}$ is more averse towards imprecision than $\succeq_{a}$

(ii) for all $(f,[\mathcal{P}, c]) \in \mathcal{A}_{B} \times \mathbb{S}, \Pi_{a}(f,[\mathcal{P}, c]) \geq \Pi_{b}(f,[\mathcal{P}, c])$

(iii) for all $[\mathcal{P}, c] \in \mathbb{S}, \mathcal{F}_{[\mathcal{P}, c]}^{a} \subseteq \mathcal{F}_{[\mathcal{P}, c]}^{b}$ 
This theorem provides an easy way of comparing two decision makers in terms of their attitudes towards imprecision: the decision maker operating the largest contraction on the set of compatible priors is the one who is the less averse towards imprecision.

\section{Discussion}

We discuss first the relationship with the literature and then offer some concluding comments.

\subsection{Relationship with the literature}

The decision criterion we axiomatized belongs to the family of "maxmin expected utility" criterion à la Gilboa and Schmeidler (1989), for which an act $f$ is preferred to an act $g$ if and only if

$\min _{p \in \mathcal{Q}} \int u \circ f d p \geq \min _{p \in \mathcal{Q}} \int u \circ g d p$, where $\mathcal{Q}$ is a revealed set of priors. The interpretation of the criterion is however somewhat different. In Gilboa and Schmeidler (1989), the information the decision maker might have is not explicitly described and the subjective set of prior $\mathcal{Q}$ is unrestricted. Thus, interpreting the min operator as reflecting "extreme pessimism" relies on a confusion. Of course, this confusion does not arise in Gilboa and Schmeidler (1989) or the subsequent specialized literature on uncertainty or ambiguity aversion (Epstein (1999), Epstein and Zhang (2001), Ghirardato and Marinacci (2002) for instance). But it is often done when applying this criterion to problems in which data are available and, without caution, the subjective family is implicitly assumed to be equal to the "objective" family, and is then used to disregard the maxmin criterion as overly pessimistic. Interestingly, Wang (2003) provides the axiomatic foundation for such an extreme pessimism in presence of objective data. His axiom of uncertainty aversion is much stronger than ours, as it compares situations that are ranked according to set inclusion. Instead, our axiom has bite only when a situation is a center preserving increase in imprecision over the other one.

Jaffray (1989) considers decision maker that have preferences over beliefs functions, rather than mere lotteries as in von Neumann and Morgenstern (1947). Imposing the independence axiom on these preferences he derives a decision criterion that is the convex combination of the minimum expected utility and the maximum expected utility. His approach however prevents a decision maker from behaving as an expected utility maximizer (i.e., as an uncertainty neutral agent).

Tapking (2003) axiomatizes a representation that is very close to ours. His decision criterion can be written as follows:

$$
f \succeq g \Leftrightarrow \int_{C} u \circ f d \nu_{(\alpha, c)} \geq \int_{C} u \circ g d \nu_{(\alpha, c)} .
$$

In this expression, $\nu_{(\alpha, c)}$ is a capacity, i.e., a set function from $\Sigma$ to $[0,1]$ and $\int_{C} u \circ f d \nu$ denotes the Choquet integral of $u \circ f$ with respect to $\nu$. Furthermore, Tapking's representation theorem 
requires that the capacity $\nu_{(\alpha, c)}$ be such that $\nu_{(\alpha, c)}(E)=\alpha \nu(E)+(1-\alpha) c(E)$ for any event $E \subset \Sigma$, where $\nu$ is a capacity, and $c$ a probability distribution.

To see the link with our decision criterion, consider a choice setting in which the informational setting $[\mathcal{P}, c]$ is fixed. Let $\nu$ be the capacity defined by: $\nu(E)=\min \{p(E) \mid p \in \operatorname{co}(\mathcal{P})\}$, for all $E \subseteq S(\mathcal{P})$. Clearly, for any $\alpha \in[0,1]$, and $c \in \mathcal{P}$, the set function $\nu_{(\alpha, c)}$ defined by $\nu_{(\alpha, c)}(E)=$ $\alpha \nu(E)+(1-\alpha) c(E)$ is a capacity with non-empty core. Therefore, in that case, the expression in Theorem 2 can be written as:

$$
\int_{C} u \circ f d \nu_{(\alpha, c)} \geq \int_{C} u \circ g d \nu_{(\alpha, c)}
$$

since the Choquet integral of $u \circ f$ with respect to a convex capacity is equal to the minimal expected utility of $f$ with respect to probability distributions in the core of the capacity (see Schmeidler (1986)). Thus, Tapking's criterion is similar to ours. However, his setup is quite different. Tapking starts with a decision maker who behaves in accordance with the Choquet expected utility model of Schmeidler (1989). Using axioms not only on a prior preference relation, but also on updated preferences, he derives an explicit definition of the capacity used by a given decision maker. This capacity is characterized by $\alpha, \nu$ and $c$, which are revealed parameters. It must be stressed that this capacity is essentially of a subjective nature, and is deduced from the decision maker behavior. Tapking (2003) furthermore provides an appealing interpretation of the revealed parameters. $c$ is interpreted as the decision maker's subjective probability judgement, whereas $\nu$ represents his subjective uncertainty. It describes the set of probability measures among which, according to the decision maker, lies the true probability measure. The size of core $(\nu)$ can therefore be seen as a measure of the confidence the decision maker's has towards his prior probability judgement. Finally, Tapking interprets the coefficient $\alpha$, as we do, i.e., as a parameter of uncertainty aversion.

Finally, we should mention Klibanoff, Marinacci, and Mukerji (2003)'s work. They, too, take the view that some piece of information may be given to the decision maker, under the form of a possible set of priors $\mathcal{P}$. They derive an axiomatic representation that can be described as follows. In order to evaluate an act, the decision maker first computes its Expected Utility for all probabilities measures in $\mathcal{P}$. Then, he computes the expectation of an increasing transformation of these evaluations, with respects to a subjective second-order probability measure over $\mathcal{P}$. In other words, the decision maker prefers an act $f$ to an act $g$ if:

$$
\mathbb{E}_{\mu} \phi\left(\mathbb{E}_{p} u \circ f\right) \geq \mathbb{E}_{\mu} \phi\left(\mathbb{E}_{p} u \circ g\right)
$$

where $\mathbb{E}$ is the expectation operator, $u$ is a von Neumann-Morgenstern utility function, $\phi$ is an increasing transformation, and $\mu$ is a subjective probability distribution over the set $\mathcal{P}$ of probability measures $p$ that are relevant. Here, $\phi$ describe the decision maker's attitude towards imprecision: it therefore plays a role similar to that of $\mathcal{F}_{[\mathcal{P}, c]}$ in our model. On the other hand, the couple $(\mathcal{P}, \mu)$ can be compared to our information $[\mathcal{P}, c]$ (although $\mu$ is of subjective nature in their model.) 


\subsection{Concluding remarks}

We have axiomatized a decision criterion that links the set of priors revealed by choice behavior in Gilboa and Schmeidler (1989) to the available information. Our approach is based on the idea that the latter can be represented through a set of priors together with a reference prior, as in Hansen and Sargent (2002) and Wang (2003). However, contrary to Wang (2003), our main axiom of aversion towards information imprecision is sufficiently weak to enable us to model a wide array of behavior, from full pessimism (maxmin over the entire set of priors) to imprecision neutrality (expected utility with respect to the reference prior). This approach, based on a description of information independent of the choice behavior of the decision maker, parallels the usual approach to risk, in which risk aversion is based on second order stochastic dominance. Our hope is that it will provide a useful benchmark in economics to pursue comparative statics exercise, in which the precision of the information is changed. Hence, the framework seems susceptible to be applied to study a wide range of questions, such as the impact of information campaign on choices, the reaction of markets after say, central bank announcements, and to provide some guidance in environmental policies or in issues like new sanitary "risks" (like the mad cow disease for instance).

Strictly speaking, our model is meant to deal with situations in which there exists an anchor which is part of the description of the information given to the decision maker. This, we argue, is representative of three types of situations: standard practice in econometrics and statistical inference (Example 3), expert committees (Example 4), sub-population and second order distribution. The three situations described can be taken as representative of situations in which there is an external criterion, to which the decision maker adheres, which determines the information he'll use. A fourth situation is when the decision maker adheres to "Laplace principle of insufficient reason". This amounts to use the uniform distribution as the center, absent any further information. For instance, in Ellsberg's three color urn example, a lot of individuals would agree that the distribution $\left(\frac{1}{3}, \frac{1}{3}, \frac{1}{3}\right)$ has a particular salience and would take this as the anchor for the set of distributions. Our model can then be used to represent these agents' preferences. However, as well-known, the principle of insufficient reason has been widely criticized. For example, assume that the decision maker knows that the exchange rate between say US-dollars and Sterling will be between $1 \mathrm{USD}=.5 £$ and $1 \mathrm{USD}=1 £$. The principle of insufficient reason would yield an "average" estimate of $1 \mathrm{USD}=.75 £$. Now, consider the alternative, equivalent, representation of the possible exchange rate by saying that the exchange rate will be between $1 £=1 \mathrm{USD}$ and $1 £=2 \mathrm{USD}$. Then, the average estimate would be $1 \mathrm{USD}=\frac{2}{3} £$. Thus, applying the principle of insufficient reason to these two logically equivalent representations of the same information leads to different averages. If one feels, with reason according to us, that this is a problem, then one should reject the use of the principle of insufficient reason and adopt an axiom à la Cohen and Jaffray (1980), stating that, in the example above, the representation of the problem should have no consequence on the choice of the agent. But this type of axiom 
prevents the decision maker from being expected utility maximizer (or even probabilistically sophisticated), and thus excludes imprecision neutrality from our setting. Another route, that we develop in ongoing research, is to address the problem directly and axiomatize a decision criterion in situations in which the available information does not allow to identify an anchor. The notion of aversion towards imprecision has then to be modified. 


\section{Appendix}

We start with an extension of the multiple prior model of Gilboa and Schmeidler (1989) taking into account the information given to the decision maker (Theorem 4 below) and then use it to prove Theorem 1. Gilboa and Schmeidler (1989) have six axioms, four of which are already included in our construction (Axioms 1, 5, 6, and 7). The other two are:

Axiom 11 (Certainty-Independence) For all $\left.f, g \in \mathcal{A}, h \in \mathcal{A}^{c},[\mathcal{P}, c] \in \mathbb{S}, \alpha \in\right] 0,1[$,

$$
(f,[\mathcal{P}, c]) \succ(g,[\mathcal{P}, c]) \Leftrightarrow(\alpha f+(1-\alpha) h,[\mathcal{P}, c]) \succ(\alpha g+(1-\alpha) h,[\mathcal{P}, c])
$$

Axiom 12 (Uncertainty aversion) For all $f, g \in \mathcal{A},[\mathcal{P}, c] \in \mathbb{S}$, and all $\alpha \in] 0,1[$,

$$
(f,[\mathcal{P}, c]) \sim(g,[\mathcal{P}, c]) \Rightarrow(\alpha f+(1-\alpha) g,[\mathcal{P}, c]) \succeq(f,[\mathcal{P}, c])
$$

Gilboa and Schmeidler have proved that Axioms 1, 5, 6, 7, 11, 12 hold if, and only if, for all $[\mathcal{P}, c]$ there exist an unique (up to a positive linear transformation) affine function $u_{[\mathcal{P}, c]}: Y \rightarrow \mathbb{R}$, and an unique, non-empty, closed and convex set $\mathcal{F}_{[\mathcal{P}, c]}$ of probability measures on $2^{S}$, such that for all $f, g \in \mathcal{A},(f,[\mathcal{P}, c]) \succeq(g,[\mathcal{P}, c])$ if and only if:

$$
\min _{p \in \mathcal{F}_{[\mathcal{P}, c]}} \int u_{[\mathcal{P}, c]} \circ f d p \geq \min _{p \in \mathcal{F}_{[\mathcal{P}, c]}} \int u_{[\mathcal{P}, c]} \circ g d p .
$$

In the next theorem, we extend the representation for variable $[\mathcal{P}, c]$.

Theorem 4 Axioms 1, 2, 5, 6, 7, 11, and 12 hold iff there exists an unique (up to a positive linear transformation) affine function $u: Y \rightarrow \mathbb{R}$, and for all $[\mathcal{P}, c] \in \mathbb{S}$, there exists a unique, non-empty, closed and convex set $\mathcal{F}_{[\mathcal{P}, c]}$ of finitely additive probability measures on $2^{S}$ such that:

1. For all $p \in \mathcal{F}_{[\mathcal{P}, c]}, p(S(\mathcal{P}))=1$,

2. For all $\varphi$ onto mapping from $S$ to $S, \mathcal{F}_{[\mathcal{P}, c]}=\left(\mathcal{F}_{[\mathcal{P}, c]}\right)^{\varphi}$,

and such that for all $f, g \in \mathcal{A},\left(f,\left[\mathcal{P}_{i}, c_{i}\right]\right) \succeq\left(g,\left[\mathcal{P}_{j}, c_{j}\right]\right)$ iff:

$$
\min _{p \in \mathcal{F}_{\left[\mathcal{P}_{i}, c_{i}\right]}} \int u \circ f d p \geq \min _{p \in \mathcal{F}_{\left[\mathcal{P}_{j}, c_{j}\right]}} \int u \circ g d p .
$$

\section{Proof. [Theorem 4]}

The necessity part of the theorem is straightforward to verify. We therefore only prove sufficiency.

Let $\left[\mathcal{P}_{i}, c_{i}\right]$ and $\left[\mathcal{P}_{j}, c_{j}\right]$ be two given situations in $\mathbb{S}$. Gilboa and Schmeidler's representation theorem implies that the decision maker is an expected utility maximizer over constant acts. 
Axiom 2 implies that $u_{\left[\mathcal{P}_{i}, c_{i}\right]}$ and $u_{\left[\mathcal{P}_{j}, c_{j}\right]}$ represent the same expected utility over constant acts (which implies that $\succeq_{\left[\mathcal{P}_{i}, c_{i}\right]}^{\ell}=\succeq_{\left[\mathcal{P}_{j}, c_{j}\right]}^{\ell}=\succeq^{\ell}$ ). Hence, they can be taken to be equal, i.e., $u_{\left[\mathcal{P}_{i}, c_{i}\right]}=$ $u_{\left[\mathcal{P}_{j}, c_{j}\right]}=u$.

To show that the representation can be extended to compare acts associated to different informational situations, let $\left(f,\left[\mathcal{P}_{i}, c_{i}\right]\right) \succeq\left(g,\left[\mathcal{P}_{j}, c_{j}\right]\right)$. Since $S\left(\mathcal{P}_{i}\right)$ and $S\left(\mathcal{P}_{j}\right)$ are finite and $f(s)$ and $g(s)$ have finite support, there exist $\bar{x}$ and $\underline{x}$ in $X$ such that for all $s \in S\left(\mathcal{P}_{i}\right) \cup S\left(\mathcal{P}_{j}\right)$, for all $x \in \operatorname{Supp}(f(s)) \cup \operatorname{Supp}(g(s)), \delta_{\bar{x}} \succeq^{\ell} \delta_{x} \succeq^{\ell} \delta_{\underline{x}}$. Hence, by Axioms 2 and 6 we know that $\left(k_{\bar{x}},\left[\mathcal{P}_{i}, c_{i}\right]\right) \succeq\left(f,\left[\mathcal{P}_{i}, c_{i}\right]\right) \succeq\left(k_{\underline{x}},\left[\mathcal{P}_{i}, c_{i}\right]\right)$ and $\left(k_{\bar{x}},\left[\mathcal{P}_{j}, c_{j}\right]\right) \succeq\left(g,\left[\mathcal{P}_{j}, c_{j}\right]\right) \succeq\left(k_{\underline{x}},\left[\mathcal{P}_{j}, c_{j}\right]\right)$ where $k_{\bar{x}}$ (resp. $k_{\underline{x}}$ ) is the constant act giving $\delta_{\bar{x}}$ (resp. $\delta_{\underline{x}}$ ) in all states. By Axioms 1 and 5 , there exists $\lambda_{i}$ such that $\left(f,\left[\mathcal{P}_{i}, c_{i}\right]\right) \sim\left(\lambda_{i} k_{\bar{x}}+\left(1-\lambda_{i}\right) k_{\underline{x}},\left[\mathcal{P}_{i}, c_{i}\right]\right)$. Similarly, there exists $\lambda_{j}$ such that $\left(g,\left[\mathcal{P}_{j}, c_{j}\right]\right) \sim\left(\lambda_{j} k_{\bar{x}}+\left(1-\lambda_{j}\right) k_{\underline{x}},\left[\mathcal{P}_{j}, c_{j}\right]\right)$. Thus,

$$
\begin{aligned}
\left(f,\left[\mathcal{P}_{i}, c_{i}\right]\right) \succeq\left(g,\left[\mathcal{P}_{j}, c_{j}\right]\right) & \Leftrightarrow\left(\lambda_{i} k_{\bar{x}}+\left(1-\lambda_{i}\right) k_{\underline{x}},\left[\mathcal{P}_{i}, c_{i}\right]\right) \succeq\left(\lambda_{j} k_{\bar{x}}+\left(1-\lambda_{j}\right) k_{\underline{x}},\left[\mathcal{P}_{j}, c_{j}\right]\right) \\
& \Leftrightarrow \lambda_{i} k_{\bar{x}}+\left(1-\lambda_{i}\right) k_{\underline{x}} \succeq^{\ell} \lambda_{j} k_{\bar{x}}+\left(1-\lambda_{j}\right) k_{\underline{x}}
\end{aligned}
$$

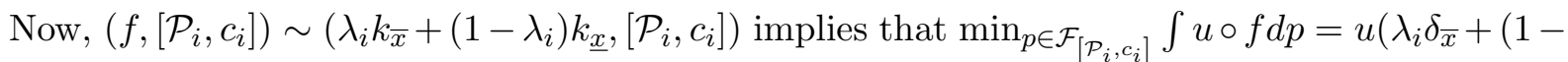

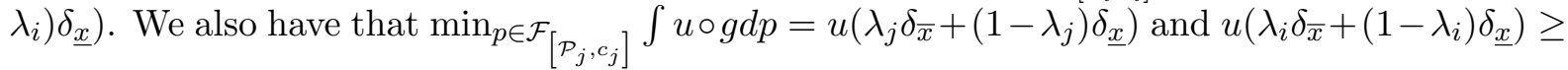
$u\left(\lambda_{j} \delta_{\bar{x}}+\left(1-\lambda_{j}\right) \delta_{\underline{x}}\right)$, which implies that

$$
\min _{p \in \mathcal{F}_{\left[\mathcal{P}_{i}, c_{i}\right]}} \int u \circ f d p \geq \min _{p \in \mathcal{F}_{\left[\mathcal{P}_{j}, c_{j}\right]}} \int u \circ g d p
$$

We now turn to Condition 1. Let $p^{*} \in \mathcal{F}_{[\mathcal{P}, c]}$ and suppose that $p^{*}(S(\mathcal{P}))=q \neq 1$. Consider $\bar{x}$ and $\underline{x}$ in $X$ such that $u\left(\delta_{\bar{x}}\right)>u\left(\delta_{\underline{x}}\right)$ and let $f$ be defined by $f(s)=\delta_{\bar{x}}$ for all $s \in S(\mathcal{P})$, $f(s)=\delta_{\underline{x}}$ for all $s \in S \backslash S(\mathcal{P})$, and $g$ by $g(s)=\delta_{\bar{x}}$ for all $s \in S$. Then

$$
\min _{p \in \mathcal{F}_{[\mathcal{P}, c]}} \int u \circ f d p \leq \int u \circ f d p^{*}=q u(\bar{x})+(1-q) u(\underline{x})<u(\bar{x})=\min _{p \in \mathcal{F}_{[\mathcal{P}, c]}} \int u \circ g d p
$$

and thus $(g,[\mathcal{P}, c]) \succ(f,[\mathcal{P}, c])$ which is a violation of Axiom 2 since $g=f_{S(\mathcal{P})} g$. Thus, for all $p \in \mathcal{F}_{[\mathcal{P}, c]}, p(S(\mathcal{P}))=1$.

We finally prove Condition 2. Let $\varphi$ be an onto mapping from $S$ to $S$ and $[\mathcal{P}, c] \in \mathbb{S}$.

We will first prove that $\mathcal{F}_{[\mathcal{P}, c]^{\varphi}} \subseteq\left(\mathcal{F}_{[\mathcal{P}, c]}\right)^{\varphi}$. Assume there exists $p^{*} \in \mathcal{F}_{[\mathcal{P}, c]^{\varphi}}$ such that $p^{*} \notin\left(\mathcal{F}_{[\mathcal{P}, c]}\right)^{\varphi}$. Since $\mathcal{F}_{[\mathcal{P}, c]}$ is a convex set, $\left(\mathcal{F}_{[\mathcal{P}, c]}\right)^{\varphi}$ is also convex. Hence, using a separation ar-

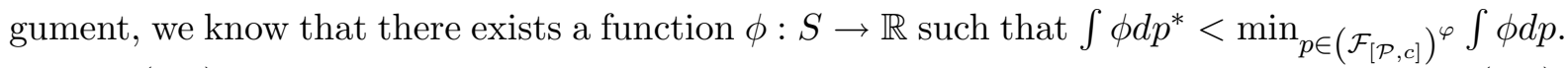
Since $S\left(\mathcal{P}^{\varphi}\right)$ is a finite set, there exist numbers $a, b$ with $a>0$, such that $\forall s \in S\left(\mathcal{P}^{\varphi}\right)$, $(a \phi(s)+b) \in u(Y)$. Then, for all $s \in S\left(\mathcal{P}^{\varphi}\right)$ there exists $y(s) \in Y$ such that $u(y(s))=a \phi(s)+b$. Define $f$ by $f(s)=y(s)$ for all $s \in S\left(\mathcal{P}^{\varphi}\right), f(s)=\delta_{x}$ for all $s \in S \backslash S\left(\mathcal{P}^{\varphi}\right)$, where $x \in X$. Define $g$ by $g^{\varphi}=f$. Since for all $p \in \mathcal{F}_{[\mathcal{P}, c]}, \int u \circ g d p=\int u \circ g^{\varphi} d p^{\varphi}$, we have:

$$
\min _{p \in \mathcal{F}_{[\mathcal{P}, c]}} \int u \circ g d p=\min _{p \in\left(\mathcal{F}_{[\mathcal{P}, c]}\right)^{\varphi}} \int u \circ g^{\varphi} d p=\min _{p \in\left(\mathcal{F}_{[\mathcal{P}, c]}\right)^{\varphi}} \int u \circ f d p
$$




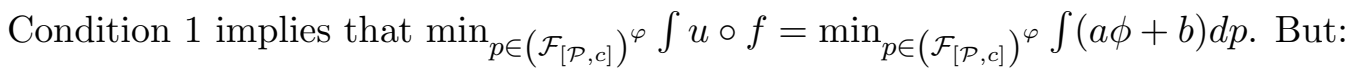

$$
\min _{p \in\left(\mathcal{F}_{[\mathcal{P}, c]}\right)^{\varphi}} \int(a \phi+b) d p>\int(a \phi+b) d p^{*} \geq \min _{p \in \mathcal{F}_{[\mathcal{P}, c]}^{\varphi}} \int u \circ f d p
$$

and therefore $(g,[\mathcal{P}, c]) \succ\left(f,[\mathcal{P}, c]^{\varphi}\right)$ which is a violation of Axiom 2 .

A similar argument may be use to prove that $\mathcal{F}_{[\mathcal{P}, c]^{\varphi}} \supseteq\left(\mathcal{F}_{[\mathcal{P}, c]}\right)^{\varphi}$.

Lemma 1 Let $[\mathcal{P}, c] \in \mathbb{S}$ and $\alpha \in[0,1]$. Let $\varphi$ be a bijection from $S(\mathcal{P})$ to $S^{\prime}$, with $S^{\prime} \cap S(\mathcal{P})=\emptyset$. Then, $[\mathcal{P}, c]_{M}^{(\alpha, \varphi)}$ is a center preserving increase in imprecision of $[\mathcal{P}, c]_{R}^{(\alpha, \varphi)}$

Proof. [Lemma 1] Straightforward

Lemma 2 Assume that Axioms 2 and 3 hold. Let $[\mathcal{P}, c] \in \mathbb{S}, f, g \in \mathcal{A}, S^{\prime} \subset \mathbb{N}$ such that $S^{\prime} \cap S(\mathcal{P})=\emptyset$, and $\varphi$ a bijection from $S$ to $S$ such that $\varphi(S(\mathcal{P}))=S^{\prime}$. Then,

$$
\forall \alpha \in[0,1], \quad(f,[\mathcal{P}, c]) \sim(g,[\mathcal{P}, c]) \Rightarrow(f,[\mathcal{P}, c]) \sim(g,[\mathcal{P}, c]) \sim\left(f_{S(\mathcal{P})} g^{\varphi},[\mathcal{P}, c]_{M}^{(\alpha, \varphi)}\right)
$$

Proof. [Lemma 2] Let $(f,[\mathcal{P}, c])$ and $(g,[\mathcal{P}, c])$ be given, such that $(f,[\mathcal{P}, c]) \sim(g,[\mathcal{P}, c])$. Without loss of generality, let $S(\mathcal{P})=\{1, \ldots, n\}$.

Consider the bijection $\varphi: S \rightarrow S$ such that $\varphi(s)=s+n$ for all $s \in\{1, \ldots, n\}, \varphi(s)=s-n$ for all $s \in\{n+1, \ldots, 2 n\}$ and $\varphi(s)=s$ for all $s>2 n$.

Axiom 2 implies: $(g,[\mathcal{P}, c]) \sim\left(g_{S(\mathcal{P})} g^{\varphi},[\mathcal{P}, c]\right)$ and $(f,[\mathcal{P}, c]) \sim\left(f_{S(\mathcal{P})} g^{\varphi},[\mathcal{P}, c]\right)$. Therefore, $\left(g_{S(\mathcal{P})} g^{\varphi},[\mathcal{P}, c]\right) \sim\left(f_{S(\mathcal{P})} g^{\varphi},[\mathcal{P}, c]\right)$. Since $S(\mathcal{P}) \cap S\left(\mathcal{P}^{\varphi}\right)=\emptyset$, and $\left(g_{S(\mathcal{P})} g^{\varphi}\right)(s)=\left(f_{S(\mathcal{P})} g^{\varphi}\right)(s)$ for all $s \in S\left(\mathcal{P}^{\varphi}\right)$, Axiom 3 implies that $(f,[\mathcal{P}, c]) \sim(g,[\mathcal{P}, c])$ if and only if:

$$
\left(g_{S(\mathcal{P})} g^{\varphi},[\mathcal{P}, c]_{M}^{(\alpha, \varphi)}\right) \sim\left(f_{S(\mathcal{P})} g^{\varphi},[\mathcal{P}, c]_{M}^{(\alpha, \varphi)}\right)
$$

Now, let $\psi: S \rightarrow S$ be an onto mapping such that $\psi(s)=s$ for all $s \in\{1, \ldots, n\}$, and $\psi(s)=s-n$ for all $s>n$. The act $g_{S(\mathcal{P})} g^{\varphi}$ is $\psi$-measurable. Thus Axiom 2 implies $\left(g_{S(\mathcal{P})} g^{\varphi},[\mathcal{P}, c]_{M}^{(\alpha, \varphi)}\right) \sim$ $\left(g_{S(\mathcal{P})} g^{\varphi},[\mathcal{P}, c]_{M}^{(\alpha, \varphi)}\right)^{\psi}$. But $\left([\mathcal{P}, c]_{M}^{(\alpha, \varphi)}\right)^{\psi}=[\mathcal{P}, c]$ and $\left(g_{S(\mathcal{P})} g^{\varphi}\right)^{\psi}=g(s)$ for all $s \in\{1, \ldots, n\}$. Therefore,

$$
(g,[\mathcal{P}, c]) \sim\left(g_{S(\mathcal{P})} g^{\varphi},[\mathcal{P}, c]_{M}^{(\alpha, \varphi)}\right)
$$

from which it follows: $(g,[\mathcal{P}, c]) \sim\left(f_{S(\mathcal{P})} g^{\varphi},[\mathcal{P}, c]_{M}^{(\alpha, \varphi)}\right)$, the desired result.

Lemma 3 Axioms 2, 3, 4, and 8 imply Axioms 11 and 12.

Proof. [Lemma 3] We first check Axiom 11. Let $(f,[\mathcal{P}, c]) \succ(g,[\mathcal{P}, c])$ and $h \in \mathcal{A}^{c}$. 
Let $\varphi: S \rightarrow S$ be a bijection such that $S\left(\mathcal{P}^{\varphi}\right) \cap S(\mathcal{P})=\emptyset$ and $\psi: S \rightarrow S$ be an onto mapping such that $\psi(s)=s$ for all $s \in S(\mathcal{P})$, and $(\psi \circ \varphi)(S(\mathcal{P}))=\left\{s^{*}\right\}$, with $s^{*} \in S \backslash S(\mathcal{P})$. Finally, let $p^{*}$ be the probability distribution defined by $p^{*}\left(s^{*}\right)=1$.

By Axiom 2,

$$
(\alpha f+(1-\alpha) h,[\mathcal{P}, c]) \sim\left(\alpha f_{S(\mathcal{P})} h+(1-\alpha) h,[\mathcal{P}, c]\right)
$$

Since $\left(\alpha f_{S(\mathcal{P})} h\right)(s)=h(s)$ for all $s \in S \backslash S(\mathcal{P})$, Axiom 4 implies:

$$
\left(\alpha f_{S(\mathcal{P})} h+(1-\alpha) h,[\mathcal{P}, c]\right) \sim\left(f_{S(\mathcal{P})} h^{\varphi},[\mathcal{P}, c]_{R}^{(\alpha, \varphi)}\right)
$$

Therefore,

$$
(\alpha f+(1-\alpha) h,[\mathcal{P}, c]) \sim\left(f_{S(\mathcal{P})} h^{\varphi},[\mathcal{P}, c]_{R}^{(\alpha, \varphi)}\right)
$$

Since $f_{S(\mathcal{P})} h^{\varphi}$ is $\psi$-measurable, Axiom 2 implies:

$$
\left(f_{S(\mathcal{P})} h^{\varphi},[\mathcal{P}, c]_{R}^{(\alpha, \varphi)}\right) \sim\left(f_{S(\mathcal{P})} h^{\varphi},[\mathcal{P}, c]_{R}^{(\alpha, \varphi)}\right)^{\psi}
$$

and therefore:

$$
(\alpha f+(1-\alpha) h,[\mathcal{P}, c]) \sim\left(f_{S(\mathcal{P})} h^{\varphi},[\mathcal{P}, c]_{R}^{(\alpha, \varphi)}\right)^{\psi}
$$

The same reasoning holds with $g$ instead of $f$, and therefore we also have:

$$
(\alpha g+(1-\alpha) h,[\mathcal{P}, c]) \sim\left(g_{S(\mathcal{P})} h^{\varphi},[\mathcal{P}, c]_{R}^{(\alpha, \varphi)}\right)^{\psi}
$$

On the other hand, by Axiom 2,

$$
(f,[\mathcal{P}, c]) \sim\left(\left(f_{S(\mathcal{P})} h^{\varphi}\right)^{\psi},[\mathcal{P}, c]\right) \text { and }(g,[\mathcal{P}, c]) \sim\left(\left(g_{S(\mathcal{P})} h^{\varphi}\right)^{\psi},[\mathcal{P}, c]\right)
$$

Therefore, $(f,[\mathcal{P}, c]) \succ(g,[\mathcal{P}, c])$ if and only if:

$$
\left(\left(f_{S(\mathcal{P})} h^{\varphi}\right)^{\psi},[\mathcal{P}, c]\right) \succ\left(\left(g_{S(\mathcal{P})} h^{\varphi}\right)^{\psi},[\mathcal{P}, c]\right)
$$

Since $\left(f_{S(\mathcal{P})} h\right)^{\psi}(s)=\left(g_{S(\mathcal{P})} h^{\varphi}\right)^{\psi}(s)$ for all $s \in S \backslash S(\mathcal{P})$ and $s^{*} \in S \backslash S(\mathcal{P})$, Axiom 3 implies that $(f,[\mathcal{P}, c]) \succ(g,[\mathcal{P}, c])$ if and only if:

$$
\left(\left(f_{S(\mathcal{P})} h^{\varphi}\right)^{\psi}, \alpha[\mathcal{P}, c]+(1-\alpha)\left[\left\{p^{*}\right\}, p^{*}\right]\right) \succ\left(\left(g_{S(\mathcal{P})} h^{\varphi}\right)^{\psi}, \alpha[\mathcal{P}, c]+(1-\alpha)\left[\left\{p^{*}\right\}, p^{*}\right]\right)
$$

But observe that $\left([\mathcal{P}, c]_{R}^{(\alpha, \varphi)}\right)^{\psi}=\alpha[\mathcal{P}, c]+(1-\alpha)\left[\left\{p^{*}\right\}, p^{*}\right]$. Therefore, equation (3) is equivalent to:

$$
\left(f_{S(\mathcal{P})} h^{\varphi},[\mathcal{P}, c]_{R}^{(\alpha, \varphi)}\right)^{\psi} \succ\left(g_{S(\mathcal{P})} h^{\varphi},[\mathcal{P}, c]_{R}^{(\alpha, \varphi)}\right)^{\psi}
$$

Finally, substituting equations (1) and (2) in (4), we obtain that $(f,[\mathcal{P}, c]) \succ(g,[\mathcal{P}, c])$ if and only if:

$$
(\alpha f+(1-\alpha) h,[\mathcal{P}, c]) \succ(\alpha g+(1-\alpha) h,[\mathcal{P}, c])
$$


thus proving Axiom 11.

We now check that Axiom 12 holds as well. Let $(f,[\mathcal{P}, c])$ and $(g,[\mathcal{P}, c])$ be given, such that $(f,[\mathcal{P}, c]) \sim(g,[\mathcal{P}, c])$. According to Lemma 2 , we have that

$$
(f,[\mathcal{P}, c]) \sim(g,[\mathcal{P}, c]) \sim\left(f_{S(\mathcal{P})} g^{\varphi},[\mathcal{P}, c]_{M}^{(\alpha, \varphi)}\right)
$$

In Lemma 1, it was shown that $[\mathcal{P}, c]_{M}^{(\alpha, \varphi)}$ was more imprecise than $[\mathcal{P}, c]_{R}^{(\alpha, \varphi)}$. Thus Axiom 8 implies that

$$
\left(f_{S(\mathcal{P})} g^{\varphi},[\mathcal{P}, c]_{R}^{(\alpha, \varphi)}\right) \succeq\left(f_{S(\mathcal{P})} g^{\varphi},[\mathcal{P}, c]_{M}^{(\alpha, \varphi)}\right)
$$

Since by Axiom 4, we have that $(\alpha f+(1-\alpha) g,[\mathcal{P}, c]) \sim\left(f_{S(\mathcal{P})} g^{\varphi},[\mathcal{P}, c]_{R}^{(\alpha, \varphi)}\right)$, it follows that

$$
(\alpha f+(1-\alpha) g,[\mathcal{P}, c]) \succeq(f,[\mathcal{P}, c]) \sim(g,[\mathcal{P}, c])
$$

Hence, Axiom 12 is satisfied.

We are now in a position to prove Theorem 1 .

\section{Proof. [Theorem 1]}

\section{Sufficiency}

By Lemma 3, we know that Axioms 2 to 4 and 8 imply Axioms 11 and 12 . Hence, we can invoke Theorem 4 to prove that there exists an unique (up to a positive affine transformation) affine function $u: Y \rightarrow \mathbb{R}$, and for all $\left[\mathcal{P}_{i}, c_{i}\right],\left[\mathcal{P}_{j}, c_{j}\right] \in \mathbb{S}$, there exist unique, non-empty, closed and convex sets $\mathcal{F}_{\left[\mathcal{P}_{i}, c_{i}\right]}$ and $\mathcal{F}_{\left[\mathcal{P}_{j}, c_{j}\right]}$ of finitely additive probability measures on $2^{S}$, such that for all $f, g \in \mathcal{A},\left(f,\left[\mathcal{P}_{i}, c_{i}\right]\right) \succeq\left(g,\left[\mathcal{P}_{j}, c_{j}\right]\right)$ if, and only if:

$$
\min _{p \in \mathcal{F}_{\left[\mathcal{P}_{i}, c_{i}\right]}} \int u \circ f d p \geq \min _{p \in \mathcal{F}_{\left[\mathcal{P}_{j}, c_{j}\right]}} \int u \circ g d p
$$

Furthermore, for all $\varphi$ onto mapping from $S$ to $S, \mathcal{F}_{\left[\mathcal{P}_{i}, c_{i}\right]^{\varphi}}=\left\{p^{\varphi} \mid p \in \mathcal{F}_{\left[\mathcal{P}_{i}, c_{i}\right]}\right\}$. We will now show that the Axioms imply conditions 1 to 5 of the Theorem.

\section{Condition 1}

Suppose Condition 1 does not hold, i.e., there exists $[\mathcal{P}, c] \in \mathbb{S}$ such that $\mathcal{F}_{[\mathcal{P}, c]} \nsubseteq \operatorname{co}(\mathcal{P})$. Then, there exists $p^{*} \in \mathcal{F}_{[\mathcal{P}, c]}$ such that $p^{*} \notin c o(\mathcal{P})$. Since $c o(\mathcal{P})$ is a convex set, using a separation argument, we know there exists a function $\phi: S \rightarrow \mathbb{R}$ such that $\int \phi d p^{*}<\min _{p \in \operatorname{co}(\mathcal{P})} \int \phi d p$. Since Axiom 9 implies that $(f,[\mathcal{P}, c]) \sim\left(f_{S(\mathcal{P})} g,[\mathcal{P}, c]\right)$, we have that Condition 1 in Theorem 4 holds and thus, for all $p \in \mathcal{F}_{[\mathcal{P}, c]}, p(S(\mathcal{P}))=1$. Thus $\operatorname{Supp}\left(p^{*}\right) \subseteq S(\mathcal{P})$ and since $S(\mathcal{P})$ is a finite set, there exist numbers $a, b$ with $a>0$, such that $\forall s \in S(\mathcal{P}),(a \phi(s)+b) \in u(Y)$. Then, for all $s \in S(\mathcal{P})$ there exists $y(s) \in Y$ such that $u(y(s))=a \phi(s)+b$. Define $f$ by $f(s)=y(s)$ for 
all $s \in S(\mathcal{P}), f(s)=\delta_{x}$ for all $s \in S \backslash S(\mathcal{P})$ where $x \in X$. Note that $\min _{p \in c o(\mathcal{P})} \int(a \phi+b) d p \in Y$ and thus there exists $y^{*}$ such that $u\left(y^{*}\right)=\min _{p \in c o(\mathcal{P})} \int(a \phi+b) d p$. Define $g$ by $g(s)=y^{*}$ for all $s \in S$. Observe that for all $p \in \Delta(S)$ such that $p \in \mathcal{P}$,

$$
\int u \circ f d p \geq \min _{p \in c o(\mathcal{P})} \int u \circ f d p=\min _{p \in c o(\mathcal{P})} \int(a \phi+b) d p=u\left(y^{*}\right)=\int u \circ g d p
$$

So for all $p \in \Delta(S)$ such that $p \in \mathcal{P},(f,[\{p\}, p]) \succeq(g,[\{p\}, p])$. Yet

$\min _{p \in \mathcal{F}_{[\mathcal{P}, c]}} \int u \circ f d p \leq \int u \circ f d p^{*}=\int(a \phi+b) d p<\min _{p \in c o(\mathcal{P})} \int(a \phi+b) d p=u\left(y^{*}\right)=\min _{p \in \mathcal{F}_{[\mathcal{P}, c]}} \int u \circ g d p$ and thus $(f,[\mathcal{P}, c]) \prec(g,[\mathcal{P}, c])$ which is a violation of Axiom 9 .

Condition 2

Condition 2 was proved in Theorem 4.

Condition 3

Consider $\left[\mathcal{P}_{i}, c_{i}\right],\left[\mathcal{P}_{j}, c_{j}\right] \in \mathbb{S}$ such that $S\left(\mathcal{P}_{i}\right) \cap S\left(\mathcal{P}_{j}\right)=\emptyset$ and $\alpha \in[0,1]$.

Step 1. $\quad \mathcal{F}_{\alpha\left[\mathcal{P}_{i}, c_{i}\right]+(1-\alpha)\left[\mathcal{P}_{j}, c_{j}\right]} \supseteq \alpha \mathcal{F}_{\left[\mathcal{P}_{i}, c_{i}\right]}+(1-\alpha) \mathcal{F}_{\left[\mathcal{P}_{j}, c_{j}\right]}$

Suppose that there exist $p_{i}^{*} \in \mathcal{F}_{\left[\mathcal{P}_{i}, c_{i}\right]}$ and $p_{j}^{*} \in \mathcal{F}_{\left[\mathcal{P}_{j}, c_{j}\right]}$ such that $p^{*}=\alpha p_{i}^{*}+(1-\alpha) p_{j}^{*} \notin$ $\mathcal{F}_{\alpha\left[\mathcal{P}_{i}, c_{i}\right]+(1-\alpha)\left[\mathcal{P}_{j}, c_{j}\right]}$. Since $\mathcal{F}_{\alpha\left[\mathcal{P}_{i}, c_{i}\right]+(1-\alpha)\left[\mathcal{P}_{j}, c_{j}\right]}$ is a convex set, using a separation argument,

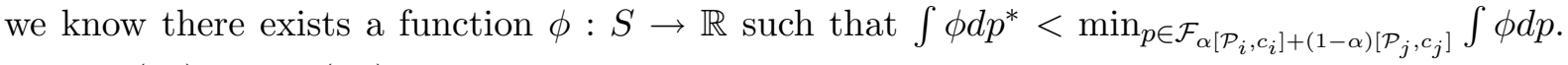
Since $S\left(\mathcal{P}_{i}\right)$ and $S\left(\mathcal{P}_{j}\right)$ are finite sets, there exist numbers $a, b$ with $a>0$, such that $\forall s \in$ $S\left(\mathcal{P}_{i}\right) \cup S\left(\mathcal{P}_{j}\right),(a \phi(s)+b) \in u(Y) .^{2} \quad$ Then, for all $s \in S\left(\mathcal{P}_{i}\right) \cup S\left(\mathcal{P}_{j}\right)$ there exists $y(s) \in$ $Y$ such that $u(y(s))=a \phi(s)+b$. Define $f$ by $f(s)=y(s)$ for all $s \in S\left(\mathcal{P}_{i}\right) \cup S\left(\mathcal{P}_{j}\right)$, $f(s)=\delta_{x}$ for all $s \in S \backslash\left(S\left(\mathcal{P}_{i}\right) \cup S\left(\mathcal{P}_{j}\right)\right)$, where $x \in X$. Since for all $p \in \mathcal{F}_{\alpha\left[\mathcal{P}_{i}, c_{i}\right]+(1-\alpha)\left[\mathcal{P}_{j}, c_{j}\right]}$, $p\left(S\left(\alpha \mathcal{P}_{i}+(1-\alpha) \mathcal{P}_{j}\right)\right)=p\left(S\left(\mathcal{P}_{i}\right) \cup S\left(\mathcal{P}_{j}\right)\right)=1$,

$$
\begin{aligned}
\min _{p \in \mathcal{F}_{\alpha\left[\mathcal{P}_{i}, c_{i}\right]+(1-\alpha)\left[\mathcal{P}_{j}, c_{j}\right]}} \int u \circ f d p & =\underset{p \in \mathcal{F}_{\alpha\left[\mathcal{P}_{i}, c_{i}\right]+(1-\alpha)\left[\mathcal{P}_{j}, c_{j}\right]}}{\min } \int(a \phi+b) d p \\
& >\int(a \phi+b) d p^{*}=\alpha \int u \circ f d p_{i}^{*}+(1-\alpha) \int u \circ f d p_{j}^{*}
\end{aligned}
$$

Since $\int u \circ f d p_{i}^{*} \in u(Y)$ and $\int u \circ f d p_{j}^{*} \in u(Y)$ there exist $y_{i}, y_{j} \in Y$ such that $u\left(y_{i}\right)=$ $\int u \circ f d p_{i}^{*}$ and $u\left(y_{j}\right)=\int u \circ f d p_{j}^{*}$. Define $g$ by $g(s)=y_{i}$ for all $s \in S\left(\mathcal{P}_{i}\right), g(s)=y_{j}$ for all $s \in S \backslash S\left(\mathcal{P}_{i}\right)$. Since for all $p \in \mathcal{F}_{\left[\mathcal{P}_{i}, c_{i}\right]}, p\left(S\left(\mathcal{P}_{i}\right)\right)=1$ we have that

$$
\min _{p \in \mathcal{F}_{\left[\mathcal{P}_{i}, c_{i}\right]}} \int u \circ g d p=u\left(y_{i}\right)=\int u \circ f d p_{i}^{*} \geq \min _{p \in \mathcal{F}_{\left[\mathcal{P}_{i}, c_{i}\right]}} \int u \circ f d p
$$

and

$$
\min _{p \in \mathcal{F}_{\left[\mathcal{P}_{j}, c_{j}\right]}} \int u \circ g d p=u\left(y_{j}\right)=\int u \circ f d p_{j}^{*} \geq \min _{\left.p \in \mathcal{F}_{\left[\mathcal{P}_{j}, c_{j}\right]}\right]} \int u \circ f d p
$$

\footnotetext{
${ }^{2}$ Completeness and continuity imply that $u(Y)$ is convex.
} 
thus $\left(g,\left[\mathcal{P}_{i}, c_{i}\right]\right) \succeq\left(f,\left[\mathcal{P}_{i}, c_{i}\right]\right)$ and $\left(g,\left[\mathcal{P}_{j}, c_{j}\right]\right) \succeq\left(f,\left[\mathcal{P}_{j}, c_{j}\right]\right)$. Furthermore, Axiom 2 implies:

$$
\begin{gathered}
\left(g,\left[\mathcal{P}_{i}, c_{i}\right]\right) \succeq\left(f_{S\left(\mathcal{P}_{i}\right)} g,\left[\mathcal{P}_{i}, c_{i}\right]\right) \sim\left(f,\left[\mathcal{P}_{i}, c_{i}\right]\right) \\
\left(f_{S\left(\mathcal{P}_{j}\right)} g,\left[\mathcal{P}_{j}, c_{j}\right]\right) \sim\left(g,\left[\mathcal{P}_{j}, c_{j}\right]\right) \succeq\left(f,\left[\mathcal{P}_{j}, c_{j}\right]\right)
\end{gathered}
$$

But by Axiom 3, equation (6) implies:

$$
\left(g, \alpha\left[\mathcal{P}_{i}, c_{i}\right]+(1-\alpha)\left[\mathcal{P}_{j}, c_{j}\right]\right) \succeq\left(f_{S\left(\mathcal{P}_{i}\right)} g, \alpha\left[\mathcal{P}_{i}, c_{i}\right]+(1-\alpha)\left[\mathcal{P}_{j}, c_{j}\right]\right)
$$

Similarly, equation (7) implies:

$$
\left(f_{S\left(\mathcal{P}_{i}\right)} g, \alpha\left[\mathcal{P}_{i}, c_{i}\right]+(1-\alpha)\left[\mathcal{P}_{j}, c_{j}\right]\right) \succeq\left(f, \alpha\left[\mathcal{P}_{i}, c_{i}\right]+(1-\alpha)\left[\mathcal{P}_{j}, c_{j}\right]\right)
$$

Therefore,

$$
\left(g, \alpha\left[\mathcal{P}_{i}, c_{i}\right]+(1-\alpha)\left[\mathcal{P}_{j}, c_{j}\right]\right) \succeq\left(f, \alpha\left[\mathcal{P}_{i}, c_{i}\right]+(1-\alpha)\left[\mathcal{P}_{j}, c_{j}\right]\right)
$$

On the other hand, since

$$
\alpha \int u \circ f d p_{i}^{*}+(1-\alpha) \int u \circ f d p_{j}^{*}=\alpha u\left(y_{i}\right)+(1-\alpha) u\left(y_{j}\right)=\int u \circ g d\left(\alpha c_{i}+(1-\alpha) c_{j}\right)
$$

equation (5) implies:

$$
\left(f, \alpha\left[\mathcal{P}_{i}, c_{i}\right]+(1-\alpha)\left[\mathcal{P}_{j}, c_{j}\right]\right) \succ\left(g,\left[\left\{\alpha c_{i}+(1-\alpha) c_{j}\right\}, \alpha c_{i}+(1-\alpha) c_{j}\right]\right)
$$

But $\alpha\left[\mathcal{P}_{i}, c_{i}\right]+(1-\alpha)\left[\mathcal{P}_{j}, c_{j}\right]$ is a center preserving increase in imprecision of $\left[\left\{\alpha c_{i}+(1-\right.\right.$ $\left.\left.\alpha) c_{j}\right\}, \alpha c_{i}+(1-\alpha) c_{j}\right]$. Therefore, Axiom 8 implies that $\left(g,\left[\left\{\alpha c_{i}+(1-\alpha) c_{j}\right\}, \alpha c_{i}+\left(1-\alpha c_{j}\right)\right]\right) \succeq$ $\left(g, \alpha\left[\mathcal{P}_{i}, c_{i}\right]+(1-\alpha)\left[\mathcal{P}_{j}, c_{j}\right]\right)$ and hence:

$$
\left(f, \alpha\left[\mathcal{P}_{i}, c_{i}\right]+(1-\alpha)\left[\mathcal{P}_{j}, c_{j}\right]\right) \succ\left(g, \alpha\left[\mathcal{P}_{i}, c_{i}\right]+(1-\alpha)\left[\mathcal{P}_{j}, c_{j}\right]\right)
$$

a contradiction with equation (8).

Step 2. $\mathcal{F}_{\alpha\left[\mathcal{P}_{i}, c_{i}\right]+(1-\alpha)\left[\mathcal{P}_{j}, c_{j}\right]} \subseteq \alpha \mathcal{F}_{\left[\mathcal{P}_{i}, c_{i}\right]}+(1-\alpha) \mathcal{F}_{\left[\mathcal{P}_{j}, c_{j}\right]}$

Suppose that there exists $p^{*} \in \mathcal{F}_{\alpha\left[\mathcal{P}_{i}, c_{i}\right]+(1-\alpha)\left[\mathcal{P}_{j}, c_{j}\right]}$ such that $p^{*} \notin \alpha \mathcal{F}_{\left[\mathcal{P}_{i}, c_{i}\right]}+(1-\alpha) \mathcal{F}_{\left[\mathcal{P}_{j}, c_{j}\right]}$.

We first show that $p^{*}\left(S\left(\mathcal{P}_{i}\right)\right)=\alpha$. Assume that such is not the case, for instance, $p^{*}\left(S\left(\mathcal{P}_{i}\right)\right)>$ $\alpha$. There exist $y_{1}, y_{2}, y_{3} \in Y$ such that $\alpha u\left(y_{1}\right)+(1-\alpha) u\left(y_{2}\right)=u\left(y_{3}\right) \cdot{ }^{3}$ Define $f$ by $f(s)=y_{1}$ for all $s \in S\left(\mathcal{P}_{i}\right), f(s)=y_{2}$ for all $s \in S \backslash S\left(\mathcal{P}_{i}\right)$ and $g$ by $g(s)=y_{3}$ for all $s \in S$. Then

$$
\begin{aligned}
& \min _{p \in \mathcal{F}_{\alpha\left[\mathcal{P}_{i}, c_{i}\right]+(1-\alpha)\left[\mathcal{P}_{j}, c_{j}\right]} \int u \circ f d p} \leq \int u \circ f d p^{*} \\
&<\alpha u\left(y_{1}\right)+(1-\alpha) u\left(y_{2}\right) \\
&=u\left(y_{3}\right)=\min _{p \in \mathcal{F}_{\alpha\left[\mathcal{P}_{i}, c_{i}\right]+(1-\alpha)\left[\mathcal{P}_{j}, c_{j}\right]} \int u \circ g d p}
\end{aligned}
$$

and thus

$$
\left(f, \alpha\left[\mathcal{P}_{i}, c_{i}\right]+(1-\alpha)\left[\mathcal{P}_{j}, c_{j}\right]\right) \prec\left(g, \alpha\left[\mathcal{P}_{i}, c_{i}\right]+(1-\alpha)\left[\mathcal{P}_{j}, c_{j}\right]\right)
$$

\footnotetext{
${ }^{3}$ Existence can be proved by using Axioms 1, 5, 7 .
} 
Without loss of generality, let $S\left(\mathcal{P}_{i}\right)=\left\{1, \ldots, n_{1}\right\}$ and $S\left(\mathcal{P}_{j}\right)=\left\{n_{1}+1, \ldots, n_{1}+n_{2}\right\}$. Consider the onto mapping $\psi: S \rightarrow S$ such that $\psi(s)=1$ for all $s \in\left\{1, \ldots, n_{1}\right\}, \psi(s)=2$ for all $s \in\left\{n_{1}+1, \ldots, n_{1}+n_{2}\right\}, \psi(s)=s+2-\left(n_{1}+n_{2}\right)$ for all $s>n_{1}+n_{2}$. Since $f$ is $\psi$-measurable, Axiom 2 implies that $\left(f, \alpha\left[\mathcal{P}_{i}, c_{i}\right]+(1-\alpha)\left[\mathcal{P}_{j}, c_{j}\right]\right) \sim\left(f, \alpha\left[\mathcal{P}_{i}, c_{i}\right]+(1-\alpha)\left[\mathcal{P}_{j}, c_{j}\right]\right)^{\psi}$. Observe that $\left(\alpha\left[\mathcal{P}_{i}, c_{i}\right]+(1-\alpha)\left[\mathcal{P}_{j}, c_{j}\right]\right)^{\psi}=\{\bar{p}\}$ where $\bar{p} \in \Delta(S)$ is such that $\bar{p}(1)=\alpha$ and $\bar{p}(2)=1-\alpha$. Therefore, $\left(f, \alpha\left[\mathcal{P}_{i}, c_{i}\right]+(1-\alpha)\left[\mathcal{P}_{j}, c_{j}\right]\right) \sim\left(f^{\psi},[\{\bar{p}\}, \bar{p}]\right)$. Note also that $[\{\bar{p}\}, \bar{p}]$ is the $(\alpha, \varphi)-$ replication of $\left[\left\{\delta_{1}\right\}, \delta_{1}\right]$ with $\varphi$ the bijection such that $\varphi(1)=2, \varphi(2)=1, \varphi(s)=s$ for all $s>2$. Define $h$ by $h(s)=\alpha y_{1}+(1-\alpha) y_{2}$ for all $s \in S$.

By Axiom 2, $\left(f^{\psi},[\{\bar{p}\}, \bar{p}]\right) \sim\left(\left(f^{\psi}\right)_{\{1,2\}} h,[\{\bar{p}\}, \bar{p}]\right)$. By Axiom 4, $\left(\left(f^{\psi}\right)_{\{1,2\}} h,[\{\bar{p}\}, \bar{p}]\right) \sim$

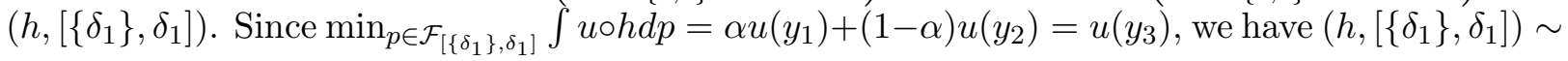
$\left(g, \alpha\left[\mathcal{P}_{i}, c_{i}\right]+(1-\alpha)\left[\mathcal{P}_{j}, c_{j}\right]\right)$. Thus we obtain

$$
\left(f, \alpha\left[\mathcal{P}_{i}, c_{i}\right]+(1-\alpha)\left[\mathcal{P}_{j}, c_{j}\right]\right) \sim\left(g, \alpha\left[\mathcal{P}_{i}, c_{i}\right]+(1-\alpha)\left[\mathcal{P}_{j}, c_{j}\right]\right)
$$

which contradicts equation (9).

Therefore, $p^{*}\left(S\left(\mathcal{P}_{i}\right)\right)=\left(1-p^{*}\left(S\left(\mathcal{P}_{j}\right)\right)=\alpha\right.$. Define $p_{k}^{*} \in \Delta(S)$ by $p_{k}^{*}(s)=\frac{p^{*}(s)}{p^{*}\left(S\left(\mathcal{P}_{k}\right)\right)}$ if $s \in S\left(\mathcal{P}_{k}\right), p_{k}^{*}(s)=0$ otherwise. There exists $k \in\{i, j\}$ such that $p_{k}^{*} \notin \mathcal{F}_{\left[\mathcal{P}_{k}, c_{k}\right]}$ (otherwise, we would have $\left.p^{*} \in \alpha \mathcal{F}_{\left[\mathcal{P}_{i}, c_{i}\right]}+(1-\alpha) \mathcal{F}_{\left[\mathcal{P}_{j}, c_{j}\right]}\right)$. Suppose for instance that $p_{i}^{*} \notin \mathcal{F}_{\left[\mathcal{P}_{i}, c_{i}\right]}$. Since $\mathcal{F}_{\left[\mathcal{P}_{i}, c_{i}\right]}$ is a convex set, using a separation argument, we know there exists a function $\phi: S \rightarrow \mathbb{R}$ such

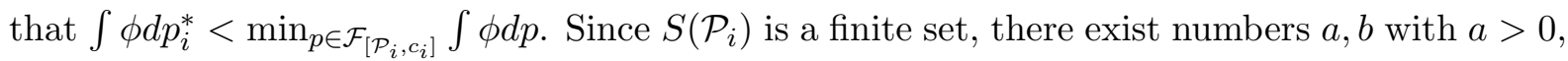
such that $\forall s \in S\left(\mathcal{P}_{i}\right),(a \phi(s)+b) \in u(Y)$. Then, for all $s \in S\left(\mathcal{P}_{i}\right)$ there exists $y(s) \in Y$ such that $u(y(s))=a \phi(s)+b$.

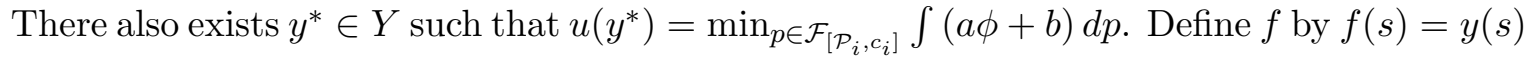
for all $s \in S\left(\mathcal{P}_{i}\right), f(s)=y^{*}$ for all $s \in S \backslash S\left(\mathcal{P}_{i}\right)$ and define $g$ by $g(s)=y^{*}$ for all $s \in S$. Since Condition 1 applies, we have that

$$
\begin{aligned}
\min _{p \in \mathcal{F}_{\left[\mathcal{P}_{i}, c_{i}\right]}} \int u \circ f d p & =\min _{p \in \mathcal{F}_{\left[\mathcal{P}_{j}, c_{j}\right]}} \int u \circ f d p \\
\min _{p \in \mathcal{F}_{\left[\mathcal{P}_{i}, c_{i}\right]}} \int u \circ g d p & =\min _{p \in \mathcal{F}_{\left[\mathcal{P}_{j}, c_{j}\right]}} \int u \circ g d p=u\left(y^{*}\right)
\end{aligned}
$$

$\operatorname{Thus}\left(f,\left[\mathcal{P}_{i}, c_{i}\right]\right) \sim\left(g,\left[\mathcal{P}_{i}, c_{i}\right]\right) \sim\left(f,\left[\mathcal{P}_{j}, c_{j}\right]\right) \sim\left(g,\left[\mathcal{P}_{j}, c_{j}\right]\right)$. By Axiom $2,\left(f,\left[\mathcal{P}_{i}, c_{i}\right]\right) \sim\left(f_{S\left(\mathcal{P}_{i}\right)} g,\left[\mathcal{P}_{i}, c_{i}\right]\right)$ By Axiom 3,

$$
\left(f_{S\left(\mathcal{P}_{i}\right)} g, \alpha\left[\mathcal{P}_{i}, c_{i}\right]+(1-\alpha)\left[\mathcal{P}_{j}, c_{j}\right]\right) \sim\left(f, \alpha\left[\mathcal{P}_{i}, c_{i}\right]+(1-\alpha)\left[\mathcal{P}_{j}, c_{j}\right]\right)
$$

and

$$
\left(f_{S\left(\mathcal{P}_{i}\right)} g, \alpha\left[\mathcal{P}_{i}, c_{i}\right]+(1-\alpha)\left[\mathcal{P}_{j}, c_{j}\right]\right) \sim\left(g, \alpha\left[\mathcal{P}_{i}, c_{i}\right]+(1-\alpha)\left[\mathcal{P}_{j}, c_{j}\right]\right)
$$

establishing that

$$
\left(f, \alpha\left[\mathcal{P}_{i}, c_{i}\right]+(1-\alpha)\left[\mathcal{P}_{j}, c_{j}\right]\right) \sim\left(g, \alpha\left[\mathcal{P}_{i}, c_{i}\right]+(1-\alpha)\left[\mathcal{P}_{j}, c_{j}\right]\right)
$$




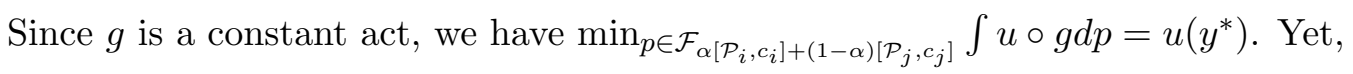

$$
\begin{aligned}
& \min _{p \in \mathcal{F}_{\alpha\left[\mathcal{P}_{i}, c_{i}\right]+(1-\alpha)\left[\mathcal{P}_{j}, c_{j}\right]} \int u \circ f d p} \leq \int u \circ f d p^{*}=\alpha \int u \circ f d p_{i}^{*}+(1-\alpha) \int u \circ f d p_{j}^{*} \\
&=\alpha \int(a \phi+b) d p_{i}^{*}+(1-\alpha) u\left(y^{*}\right) \\
&<\alpha \min _{p \in \mathcal{F}_{\left[\mathcal{P}_{i}, c_{i}\right]}} \int(a \phi+b) d p+(1-\alpha) u\left(y^{*}\right)=u\left(y^{*}\right)
\end{aligned}
$$

which contradicts equation (10).

\section{Condition 4}

Let $\left[\mathcal{P}_{i}, c_{i}\right],\left[\mathcal{P}_{j}, c_{j}\right] \in \mathbb{S}$ where $\left[\mathcal{P}_{i}, c_{i}\right]$ is a center preserving increase in imprecision of $\left[\mathcal{P}_{j}, c_{j}\right]$ (and hence $c_{i}=c_{j}$ ). Suppose that $\mathcal{F}_{\left[\mathcal{P}_{i}, c_{i}\right]} \nsupseteq \mathcal{F}_{\left[\mathcal{P}_{j}, c_{j}\right]}$ and thus, that there exists $p^{*} \in \mathcal{F}_{\left[\mathcal{P}_{j}, c_{j}\right]}$ such that $p^{*} \notin \mathcal{F}_{\left[\mathcal{P}_{i}, c_{i}\right]}$. Since $\mathcal{F}_{\left[\mathcal{P}_{i}, c_{i}\right]}$ is a convex set, using a separation argument, we know

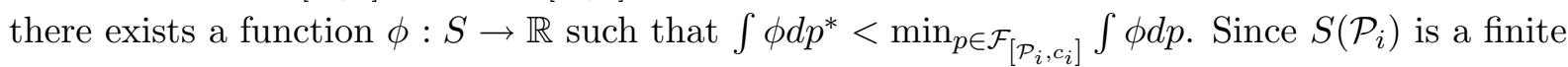
set, there exist numbers $a, b$ with $a>0$, such that $\forall s \in S\left(\mathcal{P}_{i}\right),(a \phi(s)+b) \in u(Y)$. Then, for all $s \in S\left(\mathcal{P}_{i}\right)$ there exists $y(s) \in Y$ such that $u(y(s))=a \phi(s)+b$. Define $f$ by $f(s)=y(s)$ for all $s \in S\left(\mathcal{P}_{i}\right), f(s)=\delta_{x}$ for all $s \in S \backslash S\left(\mathcal{P}_{i}\right)$, where $x \in X$. Note that since $\left[\mathcal{P}_{i}, c_{i}\right]$ is a center preserving increase in imprecision of $\left[\mathcal{P}_{j}, c_{j}\right], S\left(\mathcal{P}_{j}\right) \subseteq S\left(\mathcal{P}_{i}\right)$ and thus we have

$$
\begin{aligned}
\min _{\left.p \in \mathcal{F}_{\left[\mathcal{P}_{j}, c_{j}\right]}\right]} \int u \circ f d p & \leq \int u \circ f d p^{*}=\int(a \phi+b) d p^{*} \\
& <\min _{p \in \mathcal{F}_{\left[\mathcal{P}_{i}, c_{i}\right]}} \int(a \phi+b) d p=\min _{p \in \mathcal{F}_{\left[\mathcal{P}_{i}, c_{i}\right]} \int u \circ f d p} \int
\end{aligned}
$$

which implies that $\left(f,\left[\mathcal{P}_{i}, c_{i}\right]\right) \succ\left(f,\left[\mathcal{P}_{j}, c_{j}\right]\right)$ which is a violation of Axiom 8 .

\section{$\underline{\text { Condition } 5}$}

Let $[\mathcal{P}, c] \in \mathbb{S}$, and $[\mathcal{P}, c]_{R}^{(\alpha, \varphi)}$ a replication $(\alpha \in] 0,1[) .{ }^{4}$ We know by Lemma 1 that $[\mathcal{P}, c]_{R}^{(\alpha, \varphi)}$ is less imprecise than $[\mathcal{P}, c]_{M}^{(\alpha, \varphi)}$ and thus, since Condition 4 holds, $\mathcal{F}_{[\mathcal{P}, c]_{R}^{(\alpha, \varphi)}} \subseteq \mathcal{F}_{[\mathcal{P}, c]_{M}^{(\alpha, \varphi)}}$ and by Condition $1, \mathcal{F}_{[\mathcal{P}, c]_{R}^{(\alpha, \varphi)}}=\alpha \mathcal{F}_{[\mathcal{P}, c]}+(1-\alpha) \mathcal{F}_{[\mathcal{P}, c] \varphi}$. Thus

$$
\mathcal{F}_{[\mathcal{P}, c]_{R}^{(\alpha, \varphi)}} \subseteq \alpha \mathcal{F}_{[\mathcal{P}, c]}+(1-\alpha) \mathcal{F}_{[\mathcal{P}, c]^{\varphi}}
$$

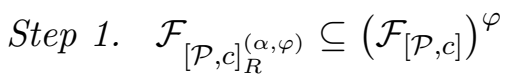

Suppose that there exists $p^{*} \in \mathcal{F}_{[\mathcal{P}, c]_{R}^{(\alpha, \varphi)}}$ such that $p^{*} \notin\left(\mathcal{F}_{[\mathcal{P}, c]}\right)^{\varphi}$. Then, since $p^{*} \in \alpha \mathcal{F}_{[\mathcal{P}, c]}+$ $(1-\alpha) \mathcal{F}_{[\mathcal{P}, c]^{\varphi}}$ and since Condition 2 holds, there exist $p_{1}^{*}, p_{2}^{*} \in \mathcal{F}_{[\mathcal{P}, c]}$ such that, $p^{*}=\alpha p_{1}^{*}+(1-$ $\alpha) p_{2}^{* \varphi}$. Note that $p_{1}^{*} \neq p_{2}^{*}$ since otherwise we would have $p^{*} \in\left(\mathcal{F}_{[\mathcal{P}, c]}\right)^{(\alpha, \varphi)}$.

Thus, there exist $E_{1}, E_{2} \subset S(\mathcal{P})$ such that $E_{1} \cap E_{2}=\emptyset, E_{1} \cup E_{2}=S(\mathcal{P}), p_{1}^{*}\left(E_{1}\right)>p_{2}^{*}\left(E_{1}\right)$ (and thus $\left.p_{1}^{*}\left(E_{2}\right)=\left(1-p_{1}^{*}\left(E_{1}\right)\right)<p_{2}^{*}\left(E_{2}\right)=\left(1-p_{1}^{*}\left(E_{2}\right)\right)\right)$. There also exist $\bar{x}$ and $\underline{x} u\left(\delta_{\bar{x}}\right)>u\left(\delta_{\underline{x}}\right)$.

\footnotetext{
${ }^{4}$ For $\alpha=0$ we have trivially $[\mathcal{P}, c]_{R}^{(0, \varphi)}=[\mathcal{P}, c]$ and for $\alpha=1$, Condition 5 can be deduced from Condition 2.
} 
Assume first that $\alpha \geq \frac{1}{2}$. Define $f$ by $f(s)=\left(\frac{2 \alpha-1}{\alpha}\right) \delta_{\bar{x}}+\left(\frac{1-\alpha}{\alpha}\right) \delta_{\underline{x}}$ for all $s \in E_{1}, f(s)=\delta_{\underline{x}}$ for all $s \in E_{2}, f(s)=\alpha \delta_{\bar{x}}+(1-\alpha) \delta_{\underline{x}}$ for all $s \in S \backslash S(\mathcal{P})$ and define $g$ by $g(s)=\delta_{\bar{x}}$ for all $s \in E_{1}$, $g(s)=\delta_{\underline{x}}$ for all $s \in E_{2}, g(s)=\alpha \delta_{\bar{x}}+(1-\alpha) \delta_{\underline{x}}$ for all $s \in S \backslash\left\{s_{1}, s_{2}\right\}$. One can easily check that $(\alpha f+(1-\alpha) g)(s)=\alpha \delta_{\bar{x}}+(1-\alpha) \delta_{\underline{x}}$ for all $s \in S(\mathcal{P})$. And thus

$$
\min _{p \in \mathcal{F}_{[\mathcal{P}, c]}} \int u \circ(\alpha f+(1-\alpha) g) d p=u\left(\alpha \delta_{\bar{x}}+(1-\alpha) \delta_{\underline{x}}\right)=\alpha u\left(\delta_{\bar{x}}\right)+(1-\alpha) u\left(\delta_{\underline{x}}\right)
$$

Consider now $f_{S(\mathcal{P})} g^{\varphi}$. We have:

$$
\begin{aligned}
\min _{p \in \mathcal{F}_{[\mathcal{P}, c]]_{R}^{(\alpha, \varphi)}}} \int u \circ f_{S(\mathcal{P})} g^{\varphi} d p & \leq \int u \circ f_{S(\mathcal{P})} g^{\varphi} d p^{*}=\alpha \int u \circ f d p_{1}^{*}+(1-\alpha) \int u \circ g d p_{2}^{*} \\
& =\alpha\left[p_{1}^{*}\left(E_{1}\right) u\left(\left(\frac{2 \alpha-1}{\alpha}\right) \delta_{\bar{x}}+\left(\frac{1-\alpha}{\alpha}\right) \delta_{\underline{x}}\right)+p_{1}^{*}\left(E_{2}\right) u\left(\delta_{\bar{x}}\right)\right] \\
& +(1-\alpha)\left[p_{2}^{*}\left(E_{1}\right) u\left(\delta_{\bar{x}}\right)+p_{2}^{*}\left(E_{2}\right) u\left(\delta_{\underline{x}}\right)\right] \\
& =\alpha u\left(\delta_{\bar{x}}\right)+(1-\alpha) u\left(\delta_{\underline{x}}\right) \\
& +(1-\alpha)\left(p_{2}^{*}\left(E_{1}\right)-p_{1}^{*}\left(E_{1}\right)\right)\left(u\left(\delta_{\bar{x}}\right)-u\left(\delta_{\underline{x}}\right)\right) \\
& <\alpha u\left(\delta_{\bar{x}}\right)+(1-\alpha) u\left(\delta_{\underline{x}}\right)
\end{aligned}
$$

and thus $(\alpha f+(1-\alpha) g,[\mathcal{P}, c]) \succ\left(f_{S(\mathcal{P})} g^{\varphi},[\mathcal{P}, c]_{R}^{(\alpha, \varphi)}\right)$ which is violation of Axiom 4 .

A similar reasoning holds in the case $\alpha \leq \frac{1}{2}$.

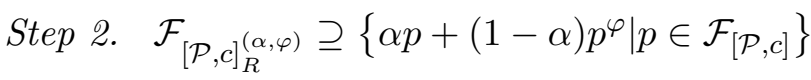

Suppose that there exists $p^{*} \in \mathcal{F}_{[\mathcal{P}, c]}$ such that $\alpha p^{*}+(1-\alpha) p^{* \varphi} \notin \mathcal{F}_{[\mathcal{P}, c]_{R}^{(\alpha, \varphi)}}$. Since we just proved that

$$
\mathcal{F}_{[\mathcal{P}, c]_{R}^{(\alpha, \varphi)}} \subseteq\left\{\alpha p+(1-\alpha) p^{\varphi} \mid p \in \mathcal{F}_{[\mathcal{P}, c]}\right\}
$$

for all $p \in \mathcal{F}_{[\mathcal{P}, c]_{R}^{(\alpha, \varphi)}}$, there exists $p_{\varphi^{-1}} \in \mathcal{F}_{[\mathcal{P}, c]}$ such that $p=\alpha p_{\varphi^{-1}}+(1-\alpha)\left(p_{\varphi^{-1}}\right)^{\varphi}$. Consider $\mathcal{F}=\left\{p_{\varphi^{-1}} \mid p \in \mathcal{F}_{[\mathcal{P}, c]_{R}^{(\alpha, \varphi)}}\right\}$. Since $\mathcal{F}_{[\mathcal{P}, c]_{R}^{(\alpha, \varphi)}}$ is convex, $\mathcal{F}$ is also convex and $p^{*} \notin \mathcal{F}$. Hence, using a separation argument, we know there exists a function $\phi: S \rightarrow \mathbb{R}$ such that $\int \phi d p^{*}<$ $\min _{p \in \mathcal{F}} \int \phi d p$. Since $S(\mathcal{P})$ is a finite set, there exist numbers $a, b$ with $a>0$, such that $\forall s \in S(\mathcal{P})$, $(a \phi(s)+b) \in u(Y)$. Then, for all $s \in S(\mathcal{P})$ there exists $y(s) \in Y$ such that $u(y(s))=a \phi(s)+b$. Define $f$ by $f(s)=y(s)$ for all $s \in S(\mathcal{P}), f(s)=\delta_{x}$ for all $s \in S \backslash S\left(\mathcal{P}^{\varphi}\right)$, where $x \in X$. Observe that for all $p \in \mathcal{F}_{[\mathcal{P}, c]_{R}^{(\alpha, \varphi)}}$,

$$
\begin{aligned}
\int u \circ f_{S(\mathcal{P})} f^{\varphi} d p & =\int u \circ f_{S(\mathcal{P})} f^{\varphi} d\left(\alpha p_{\varphi^{-1}}+(1-\alpha)\left(p_{\varphi^{-1}}\right)^{\varphi}\right) \\
& =\alpha \int u \circ f d p_{\varphi^{-1}}+(1-\alpha) \int u \circ f^{\varphi} d\left(p_{\varphi^{-1}}\right)^{\varphi} \\
& =\int u \circ f d p_{\varphi^{-1}}=\int(a \phi+b) d p_{\varphi^{-1}}
\end{aligned}
$$


Thus

$$
\begin{aligned}
\min _{p \in \mathcal{F}_{[\mathcal{P}, c]}} \int u \circ f d p & \leq \int u \circ f d p^{*}=\int(a \phi+b) d p^{*}<\min _{p \in \mathcal{F}} \int(a \phi+b) d p \\
& =\min _{p \in \mathcal{F}} \int u \circ f d p=\min _{p \in \mathcal{F}_{[\mathcal{P}, c]}(\alpha, \varphi)} \int u \circ f_{S(\mathcal{P})} f^{\varphi} d p
\end{aligned}
$$

which shows that $\left(f_{S(\mathcal{P}} f^{\varphi},[\mathcal{P}, c]_{R}^{(\alpha, \varphi)}\right) \succ(f,[\mathcal{P}, c])$, a violation of Axiom 4 .

\section{Necessity}

The axioms to check are Axioms 3, 4, 8, and 9 since the others hold by Theorem 4 .

\section{$\underline{\text { Axiom } 3}$}

Consider $\left[\mathcal{P}_{i}, c_{i}\right] \in \mathbb{S}, i=1,2,3$ such that $\left(S\left(\mathcal{P}_{1}\right) \cup S\left(\mathcal{P}_{2}\right)\right) \cap S\left(\mathcal{P}_{3}\right)=\emptyset, \quad \alpha \in[0,1]$, and $f, g \in \mathcal{A}$ such that $f(s)=g(s)$ for all $s \in S\left(\mathcal{P}_{3}\right)$. Assume $\left(f,\left[\mathcal{P}_{1}, c_{1}\right]\right) \succeq\left(g,\left[\mathcal{P}_{2}, c_{2}\right]\right)$. By Condition $3, \mathcal{F}_{\alpha\left[\mathcal{P}_{1}, c_{1}\right]+(1-\alpha)\left[\mathcal{P}_{3}, c_{3}\right]}=\alpha \mathcal{F}_{\left[\mathcal{P}_{1}, c_{1}\right]}+(1-\alpha) \mathcal{F}_{\left[\mathcal{P}_{3}, c_{3}\right]}$ we have that

$$
\begin{aligned}
\min _{p \in \mathcal{F}_{\alpha\left[\mathcal{P}_{1}, c_{1}\right]+(1-\alpha)\left[\mathcal{P}_{3}, c_{3}\right]} \int u \circ f d p} & =\min _{p \in \alpha \mathcal{F}_{\left[\mathcal{P}_{1}, c_{1}\right]}+(1-\alpha) \mathcal{F}_{\left[\mathcal{P}_{3}, c_{3}\right]}} \int u \circ f d p \\
& =\alpha \min _{p \in \mathcal{F}_{\left[\mathcal{P}_{1}, c_{1}\right]}} \int u \circ f d p+(1-\alpha) \min _{p \in \mathcal{F}_{\left[\mathcal{P}_{3}, c_{3}\right]}} \int u \circ f d p \\
& \geq \alpha \min _{p \in \mathcal{F}_{\left[\mathcal{P}_{2}, c_{2}\right]}} \int u \circ g d p+(1-\alpha) \min _{p \in \mathcal{F}_{\left[\mathcal{P}_{3}, c_{3}\right]}} \int u \circ f d p
\end{aligned}
$$

But we have also

$$
\min _{p \in \mathcal{F}_{\alpha\left[\mathcal{P}_{1}, c_{1}\right]+(1-\alpha)\left[\mathcal{P}_{3}, c_{3}\right]}} \int u \circ g d p=\alpha \min _{p \in \mathcal{F}_{\left[\mathcal{P}_{2}, c_{2}\right]}} \int u \circ g d p+(1-\alpha) \min _{p \in \mathcal{F}_{\left[\mathcal{P}_{3}, c_{3}\right]}} \int u \circ f d p
$$

thus $\left(f, \alpha\left[\mathcal{P}_{1}, c_{1}\right]+(1-\alpha)\left[\mathcal{P}_{3}, c_{3}\right]\right) \succeq\left(g, \alpha\left[\mathcal{P}_{1}, c_{1}\right]+(1-\alpha)\left[\mathcal{P}_{3}, c_{3}\right]\right)$, which proves that Axiom 3 is satisfied.

\section{$\underline{\text { Axiom } 4}$}

Consider $[\mathcal{P}, c] \in \mathbb{S}$, the replication $[\mathcal{P}, c]_{R}^{(\alpha, \varphi)}, f, g \in \mathcal{A}$ such that $f(s)=g(s)$ for all $s \in$ $S \backslash S(\mathcal{P})$. Since Condition 5 holds,

$$
\min _{p \in \mathcal{F}_{[\mathcal{P}, c]]_{R}^{(\alpha, \varphi)}}^{(\alpha, j)}} \int u \circ f_{S(\mathcal{P})} g^{\varphi} d p=\min _{p \in \mathcal{F}_{[\mathcal{P}, c]}} \int u \circ f_{S(\mathcal{P})} g^{\varphi} d\left(\alpha p+(1-\alpha) p^{\varphi}\right)
$$

For all $p \in \mathcal{F}_{[\mathcal{P}, c]}$,

$$
\begin{aligned}
\int u \circ f_{S(\mathcal{P})} g^{\varphi} d\left(\alpha p+(1-\alpha) p^{\varphi}\right) & =\alpha \int u \circ f_{S(\mathcal{P})} g^{\varphi} d p+(1-\alpha) \int u \circ f_{S(\mathcal{P})} g^{\varphi} d p^{\varphi} \\
& =\alpha \int u \circ f d p+(1-\alpha) \int u \circ g d p \\
& =\int u \circ(\alpha f+(1-\alpha) g) d p
\end{aligned}
$$


and thus

$$
\min _{p \in \mathcal{F}_{[\mathcal{P}, c]}} \int u \circ f_{S(\mathcal{P})} g^{\varphi} d\left(\alpha p+(1-\alpha) p^{\varphi}\right)=\min _{p \in \mathcal{F}_{[\mathcal{P}, c]}} \int u \circ(\alpha f+(1-\alpha) g) d p
$$

which shows that $(\alpha f+(1-\alpha) g,[\mathcal{P}, c]) \sim\left(f_{S(\mathcal{P})} g^{\varphi},[\mathcal{P}, c]_{R}^{(\alpha, \varphi)}\right)$.

$\underline{\text { Axiom } 8}$

By Condition 4 , if $\left[\mathcal{P}_{i}, c_{i}\right]$ is a center preserving increase in imprecision of $\left[\mathcal{P}_{j}, c_{j}\right]$ then $\mathcal{F}_{\left[\mathcal{P}_{i}, c_{i}\right]} \supseteq \mathcal{F}_{\left[\mathcal{P}_{j}, c_{j}\right]}$ implies that for all $f \in \mathcal{A}$,

$$
\min _{p \in \mathcal{F}_{\left[\mathcal{P}_{i}, c_{i}\right]}} \int u \circ f d p \leq \min _{p \in \mathcal{F}_{\left[\mathcal{P}_{j}, c_{j}\right]}} \int u \circ f d p
$$

and thus $\left(f,\left[\mathcal{P}_{j}, c_{j}\right]\right) \succeq\left(f,\left[\mathcal{P}_{i}, c_{i}\right]\right)$ which proves that Axiom 8 is satisfied.

$\underline{\text { Axiom } 9}$

Consider $f, g \in \mathcal{A}$ and $[\mathcal{P}, c] \in \mathbb{S}$ such that $(f,[\{p\}, p]) \succeq(g,[\{p\}, p])$ for all $p \in \mathcal{P}$. Remark that for all $p \in c o(\mathcal{P})$ there exist $p_{1}, p_{2} \in \Delta(S)$ and $\alpha \in[0,1]$ such that $p=\alpha p_{1}+(1-\alpha) p_{2}$. Thus,

$$
\begin{aligned}
\int u \circ f d\left(\alpha p_{1}+(1-\alpha) p_{2}\right) & =\alpha \int u \circ f d p_{1}+(1-\alpha) \int u \circ f d p_{2} \\
& \geq \alpha \int u \circ g d p_{1}+(1-\alpha) \int u \circ g d p_{2} \\
& =\int u \circ g d\left(\alpha p_{1}+(1-\alpha) p_{2}\right)
\end{aligned}
$$

and hence $\int u \circ f d p \geq \int u \circ g d p$.

Since by Condition $1, \mathcal{F}_{[\mathcal{P}, c]} \subseteq c o(\mathcal{P})$, for all $p \in \mathcal{F}_{[\mathcal{P}, c]}, \int u \circ f d p \geq \int u \circ g d p$ and thus $\min _{p \in \mathcal{F}_{[\mathcal{P}, c]}} \int u \circ f d p \geq \min _{p \in \mathcal{F}_{[\mathcal{P}, c]}} \int u \circ g d p$ which implies that $(f,[\mathcal{P}, c]) \succeq(g,[\mathcal{P}, c])$.

Proof. [Theorem 2] We introduce first a piece of notation. Let $[\mathcal{P}, c]$ be a situation, then $\mathcal{P}^{\alpha}=\left\{p^{\alpha} \in \Delta(S) \mid p^{\alpha}=\alpha p+(1-\alpha) c, p \in \mathcal{P}\right\} . \mathcal{P}^{\alpha}$ is the "contraction" of $\mathcal{P}$ around its anchor $c$, at rate $\alpha$. In Theorem 2, $\alpha \min _{p \in \operatorname{co}(\mathcal{P})} \int u \circ f d p+(1-\alpha) \int u \circ f d c=\min _{p \in c o\left(\mathcal{P}^{\alpha}\right)} \int u \circ f d p$.

\section{Sufficiency}

Axioms 8 and 9 are clearly satisfied. By Theorem 1, we know there exist an unique (up to a positive linear transformation) affine function $u: Y \rightarrow \mathbb{R}$, and for all $\left[\mathcal{P}_{1}, c_{1}\right],\left[\mathcal{P}_{1}, c_{1}\right] \in \mathbb{S}$, there exist unique, non-empty, closed and convex set $\mathcal{F}_{\left[\mathcal{P}_{1}, c_{1}\right]}$ and $\mathcal{F}_{\left[\mathcal{P}_{2}, c_{2}\right]}$ of probability measures on $2^{S}$, satisfying Conditions 1 to 5 of Theorem 1 and such that for all $f, g \in \mathcal{A},\left(f,\left[\mathcal{P}_{1}, c_{1}\right]\right) \succeq\left(g,\left[\mathcal{P}_{2}, c_{2}\right]\right)$ iff:

$$
\min _{p \in \mathcal{F}_{\left[\mathcal{P}_{1}, c_{1}\right]}} \int u \circ f d p \geq \min _{p \in \mathcal{F}_{\left[\mathcal{P}_{2}, c_{2}\right]}} \int u \circ g d p
$$


Step $1 . \forall\left[\mathcal{P}_{i}, c_{i}\right] \in \mathbb{S}, \exists \alpha_{i}$ s.t. $\mathcal{F}_{\left[\mathcal{P}_{i}, c_{i}\right]}=c o\left(\mathcal{P}_{i}^{\alpha_{i}}\right)$

Let $\left[\mathcal{P}_{i}, c_{i}\right] \in \mathbb{S}$. By Condition 1 of Theorem 1, we have that $\mathcal{F}_{\left[\mathcal{P}_{i}, c_{i}\right]} \subseteq \operatorname{co}\left(\mathcal{P}_{i}\right)$. On the other hand, since $\left[\mathcal{P}_{i}, c_{i}\right]$ is a center preserving increase in imprecision of $\left[\{c\}_{i}, c_{i}\right]$, Condition 4 of Theorem 1 implies that $\mathcal{F}_{\left[\left\{c_{i}\right\}, c_{i}\right]} \subseteq \mathcal{F}_{\left[\mathcal{P}_{i}, c_{i}\right]}$ and hence $c_{i} \in \mathcal{F}_{\left[\mathcal{P}_{i}, c_{i}\right]}$.

Remark also that $\operatorname{Supp}\left(c_{i}\right)=S\left(\mathcal{P}_{i}^{0}\right)$ and that for all $\beta, \gamma \in[0,1]$ such that $\beta \geq \gamma$, we have that $\operatorname{co}\left(\mathcal{P}_{i}^{\beta}\right) \supseteq \operatorname{co}\left(\mathcal{P}_{i}^{\gamma}\right)$. Let

$$
\underline{\beta}_{i}=\operatorname{Sup}\left\{\beta \mid \beta \in[0,1] \text { and } \operatorname{co}\left(\mathcal{P}_{i}^{\beta}\right) \subseteq \mathcal{F}_{\left[\mathcal{P}_{i}, c_{i}\right]}\right\}
$$

and

$$
\bar{\beta}_{i}=\operatorname{Inf}\left\{\beta \mid \beta \in[0,1] \text { and } \operatorname{co}\left(\mathcal{P}_{i}^{\beta}\right) \supseteq \mathcal{F}_{\left[\mathcal{P}_{i}, c_{i}\right]}\right\}
$$

Since for all $\beta \in[0,1], c o\left(\mathcal{P}_{i}^{\beta}\right)$ and $\mathcal{F}_{\left[\mathcal{P}_{i}, c_{i}\right]}$ are closed sets, we have that

$$
\operatorname{co}\left(\mathcal{P}_{i}^{\underline{\beta}_{i}}\right) \subseteq \mathcal{F}_{\left[\mathcal{P}_{i}, c_{i}\right]} \subseteq \operatorname{co}\left(\mathcal{P}_{i}^{\bar{\beta}_{i}}\right)
$$

Assume that $\underline{\beta}_{i}<\bar{\beta}_{i}$. Let $\left.\beta \in\right] \underline{\beta}_{i}, \bar{\beta}_{i}\left[\right.$. Then there exist $p_{1}, p_{2}$ in $\operatorname{co}\left(\mathcal{P}_{i}\right) \backslash \operatorname{Int}\left(\operatorname{co}\left(\mathcal{P}_{i}\right)\right)$ such that $\left(\beta p_{1}+(1-\beta) c\right) \in \mathcal{F}_{\left[\mathcal{P}_{i}, c_{i}\right]}$ and $\left(\beta p_{2}+(1-\beta) c_{i}\right) \notin \mathcal{F}_{\left[\mathcal{P}_{i}, c_{i}\right]}{ }^{5}$ Since $\mathcal{F}_{\left[\mathcal{P}_{i}, c_{i}\right]}$ is a convex set, using a separation argument, we know there exists a function $\phi_{2}: S \rightarrow \mathbb{R}$ such that

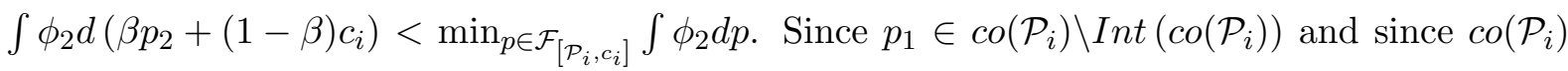
is a convex set, using a separation argument, we know there exists a function $\phi_{1}: S \rightarrow \mathbb{R}$ such that $\int \phi_{1} d p_{1} \leq \min _{p \in \operatorname{co}\left(\mathcal{P}_{i}\right)} \int \phi_{1} d p$.

Since $S\left(\mathcal{P}_{i}\right)$ is a finite set, there exist numbers $a_{i}, b_{i}$ with $a_{i}>0$, such that $\forall s \in S\left(\mathcal{P}_{i}\right)$, $\left(a_{i} \phi_{i}(s)+b_{i}\right) \in u(Y)$ for $i=1,2$,

$$
\left\{\begin{aligned}
\min _{p \in c o\left(\mathcal{P}_{i}\right)} \int\left(a_{1} \phi_{1}+b_{1}\right) d p & =\min _{p \in \operatorname{co}\left(\mathcal{P}_{i}\right)} \int\left(a_{2} \phi_{2}+b_{2}\right) d p \\
\int\left(a_{1} \phi_{1}+b_{1}\right) d c_{i} & =\int\left(a_{2} \phi_{2}+b_{2}\right) d c_{i}
\end{aligned}\right.
$$

Then, for all $s \in S\left(\mathcal{P}_{i}\right)$ there exists $y_{i}(s) \in Y$ such that $u\left(y_{i}(s)\right)=a_{i} \phi_{i}(s)+b_{i}$. Define $f$ by $f(s)=y_{1}(s)$ for all $s \in S\left(\mathcal{P}_{i}\right), f(s)=\delta_{x}$ for all $s \in S \backslash S\left(\mathcal{P}_{i}\right)$, where $x \in X$ and $g$ by $g(s)=y_{2}(s)$ for all $s \in S\left(\mathcal{P}_{i}\right), g(s)=\delta_{x}$ for all $s \in S \backslash S\left(\mathcal{P}_{i}\right)$. Thus we have

$$
\min _{p \in \operatorname{co}\left(\mathcal{P}_{i}\right)} \int u \circ f d p=\min _{p \in c o\left(\mathcal{P}_{i}\right)} \int u \circ g d p
$$

and

$$
\int u \circ f d c_{i}=\int u \circ g d c_{i}
$$

Since $\operatorname{co}\left(\mathcal{P}_{i}\right)$ is closed, there exists $p \in \operatorname{co}\left(\mathcal{P}_{i}\right)$ such that $\min _{p \in \operatorname{co}\left(\mathcal{P}_{i}\right)} \int u \circ g d p=\int u \circ g d p$. Then for all $q \in \operatorname{co}\left(\mathcal{P}_{i}\right)$, we have that $(f,[\{q\}, q]) \succeq(g,[\{p\}, p])$ and thus Axiom 10 implies that $\left(f,\left[\mathcal{P}_{i}, c_{i}\right]\right) \succeq$ $\left(g,\left[\mathcal{P}_{i}, c_{i}\right]\right)$. Similarly, $\left(g,\left[\mathcal{P}_{i}, c_{i}\right]\right) \succeq\left(f,\left[\mathcal{P}_{i}, c_{i}\right]\right)$, and therefore $\left(f,\left[\mathcal{P}_{i}, c_{i}\right]\right) \sim\left(g,\left[\mathcal{P}_{i}, c_{i}\right]\right)$.

\footnotetext{
${ }^{5}$ Such a couple $\left(p_{1}, p_{2}\right)$ exists since otherwise, we would have either $\operatorname{co}\left(\mathcal{P}_{i}^{\beta}\right) \subseteq \mathcal{F}_{\left[\mathcal{P}_{i}, c_{i}\right]}$ or $\operatorname{co}\left(\mathcal{P}_{i}^{\beta}\right) \supseteq \mathcal{F}_{\left[\mathcal{P}_{i}, c_{i}\right]}$.
} 
On the other hand

$$
\begin{aligned}
& \min _{p \in \mathcal{F}_{\left[\mathcal{P}_{i}, c_{i}\right]} \int u \circ g d p}>\int u \circ g d\left(\beta p_{2}+(1-\beta) c_{i}\right) \\
&=\beta \int u \circ g d p_{2}+(1-\beta) \int u \circ g d c_{i} \\
& \geq \beta \min _{p \in \operatorname{co}\left(\mathcal{P}_{i}\right)} \int u \circ g d p+(1-\beta) \int u \circ g d c_{i} \\
&=\beta \min _{p \in \operatorname{co}\left(\mathcal{P}_{i}\right)} \int u \circ f d p+(1-\beta) \int u \circ f d c_{i} \\
&=\beta \int u \circ f d p_{1}+(1-\beta) \int u \circ f d c_{i} \\
&=\int u \circ f d\left(\beta p_{1}+(1-\beta) c_{i}\right) \\
& \geq \min _{p \in \mathcal{F}_{\left[\mathcal{P}_{i}, c_{i}\right]}} \int u \circ f d p
\end{aligned}
$$

which yields a contradiction. Therefore, $\underline{\beta}_{i}=\bar{\beta}_{i}$, and we have $\mathcal{F}_{\left[\mathcal{P}_{i}, c_{i}\right]}=\operatorname{co}\left(\mathcal{P}_{i}^{\alpha_{i}}\right)$ with $\alpha_{i}=\underline{\beta}_{i}=$ $\bar{\beta}_{i}$.

Step 2. $\exists \alpha$ s.t. $\forall\left[\mathcal{P}_{i}, c_{i}\right] \in \mathbb{S}, \mathcal{F}_{\left[\mathcal{P}_{i}, c_{i}\right]}=\operatorname{co}\left(\mathcal{P}_{i}^{\alpha}\right)$

To simplify notation, denote $\alpha_{\gamma\left[\mathcal{P}_{i}, c_{i}\right]+(1-\gamma)\left[\mathcal{P}_{j}, c_{j}\right]}$ by $\alpha_{(\gamma, i, j)}$.

Suppose that $\alpha_{1} \neq \alpha_{2}$ for $\left[\mathcal{P}_{1}, c_{1}\right],\left[\mathcal{P}_{2}, c_{2}\right] \in \mathbb{S}$. Without loss of generality, consider that $S\left(\mathcal{P}_{1}\right) \cap S\left(\mathcal{P}_{2}\right)=\emptyset .{ }^{6}$ Let $\left.\gamma \in\right] 0,1[$. Since Condition 3 of Theorem 1 holds, we have that

$$
\mathcal{F}_{\gamma\left[\mathcal{P}_{1}, c_{1}\right]+(1-\gamma)\left[\mathcal{P}_{2}, c_{2}\right]}=\gamma \mathcal{F}_{\left[\mathcal{P}_{1}, c_{1}\right]}+(1-\gamma) \mathcal{F}_{\left[\mathcal{P}_{2}, c_{2}\right]}
$$

On the other hand,

$$
\mathcal{F}_{\gamma\left[\mathcal{P}_{1}, c_{1}\right]+(1-\gamma)\left[\mathcal{P}_{2}, c_{2}\right]}=c o\left(\gamma \mathcal{P}_{1}+(1-\gamma) \mathcal{P}_{2}\right)^{\alpha(\gamma, 1,2)}
$$

Necessarily, either $\alpha_{(\gamma, 1,2)} \neq \alpha_{1}$ or $\alpha_{(\gamma, 1,2)} \neq \alpha_{2}$ and we run into a contradiction. For instance, suppose $\alpha_{(\gamma, 1,2)}>\alpha_{1}$ and consider $p_{i} \in \operatorname{co}\left(\mathcal{P}_{i}\right) \backslash \operatorname{Int}\left(\operatorname{co}\left(\mathcal{P}_{i}\right)\right)$ for $i=1,2$. We have then:

$$
\left.\gamma\left[\alpha_{(\gamma, 1,2)} p_{1}+\left(1-\alpha_{(\gamma, 1,2)}\right) c_{1}\right)\right]+(1-\gamma)\left[\alpha_{(\gamma, 1,2)} p_{2}+\left(1-\alpha_{(\gamma, 1,2)}\right) c_{2}\right] \in \mathcal{F}_{\gamma\left[\mathcal{P}_{1}, c_{1}\right]+(1-\gamma)\left[\mathcal{P}_{2}, c_{2}\right]}
$$

and thus

$$
\left[\alpha_{(\gamma, 1,2)} p_{1}+\left(1-\alpha_{(\gamma, 1,2)}\right) c_{1}\right] \in \mathcal{F}_{\left[\mathcal{P}_{1}, c_{1}\right]}
$$

which yields a contradiction to the fact that $\alpha_{(\gamma, 1,2)}>\alpha_{1}$. The proof can be adapted to the other cases.

Step 3.

\footnotetext{
${ }^{6}$ We can always find a bijection $\varphi$ such that $S\left(\mathcal{P}_{1}\right) \cap S\left(\mathcal{P}_{2}^{\varphi}\right)=\emptyset$ and it can be easily check that since Condition 1 of Theorem 1 holds, we have that $\alpha_{\left[\mathcal{P}_{2}, c_{2}\right]}=\alpha_{\left[\mathcal{P}_{2}^{\varphi}, c_{2}^{\varphi}\right]}$.
} 
Finally, it can be easily checked that for all $\left[\mathcal{P}_{1}, c_{1}\right],\left[\mathcal{P}_{2}, c_{2}\right] \in \mathbb{S}$, for all $f, g \in \mathcal{A}$,

$$
\min _{p \in \operatorname{co}\left(\mathcal{P}_{1}^{\alpha}\right)} \int u \circ f d p=\alpha \min _{p \in \operatorname{co}\left(\mathcal{P}_{1}\right)} \int u \circ f d p+(1-\alpha) \int u \circ f d c_{1}
$$

\section{Necessity}

It is easy to check that Conditions 1 to 5 of Theorem 1 hold and thus following the previous proofs, Axioms 1 to 7 hold. We hence simply have to check Axiom 10. Consider $f, g \in \mathcal{A}$, $\left[\mathcal{P}_{1}, c_{1}\right],\left[\mathcal{P}_{2}, c_{2}\right] \in \mathbb{S}$, such that $\left(f,\left[\left\{c_{1}\right\} c_{1}\right]\right) \succeq\left(g,\left[\left\{c_{2}\right\}, c_{2}\right]\right)$ and for all $p \in \operatorname{co}\left(\mathcal{P}_{1}\right)$, there exists $q \in \operatorname{co}\left(\mathcal{P}_{2}\right)$ such that $(f,[\{p\}, p]) \succeq(g,[\{q\}, q])$.

Given the representation obtained, this implies first that

$$
\int u \circ f d c_{1} \geq \int u \circ g d c_{2}
$$

Furthermore, $\forall p \in \operatorname{co}\left(\mathcal{P}_{1}\right)$, there exists $q \in \operatorname{co}\left(\mathcal{P}_{2}\right)$ such that $\int u \circ f d p \geq \int u \circ g d q$. Hence,

$$
\min _{p \in \operatorname{co}\left(\mathcal{P}_{1}\right)} \int u \circ f d p \geq \min _{q \in \operatorname{co}\left(\mathcal{P}_{2}\right)} \int u \circ g d q
$$

Thus,

$$
\alpha \min _{p \in \operatorname{co}\left(\mathcal{P}_{1}\right)} \int u \circ f d p+(1-\alpha) \int u \circ f d c_{1} \geq \alpha \min _{p \in \operatorname{co}\left(\mathcal{P}_{2}\right)} \int u \circ f d p+(1-\alpha) \int u \circ g d c_{2}
$$

and thus $\left(f,\left[\mathcal{P}_{1}, c_{1}\right]\right) \succeq\left(g,\left[\mathcal{P}_{2}, c_{2}\right]\right)$.

\section{Proof. [Theorem 3]}

For $i \in\{a, b\}$, let $V_{i}(f,[\mathcal{P}, c])=\min _{p \in \mathcal{F}_{[\mathcal{P}, c]}^{i}} \int u_{i} \circ f d p$ (as defined in Theorem 1.)

$[(i) \Rightarrow(i i)]$

Let $(f,[\mathcal{P}, c]) \in \mathcal{A}_{B} \times \mathbb{S}$. By definition, $\left(k_{P e_{a}(f,[\mathcal{P}, c])},[\mathcal{P}, c]\right) \sim_{a}(f,[\mathcal{P}, c])$. Since $\succeq_{b}$ is more uncertainty averse than $\succeq_{a}$, one has: $\left(k_{P e_{a}(f,[\mathcal{P}, c])},[\mathcal{P}, c]\right) \succeq_{b}(f,[\mathcal{P}, c])$, and, by definition, $(f,[\mathcal{P}, c]) \sim_{b}\left(k_{P e_{b}(f,[\mathcal{P}, c])},[\mathcal{P}, c]\right)$. Therefore, $\left(k_{P e_{a}(f,[\mathcal{P}, c])},[\mathcal{P}, c]\right) \succeq_{b}\left(k_{P e_{b}(f,[\mathcal{P}, c])},[\mathcal{P}, c]\right)$. Hence, $u_{a}\left(k_{P e_{a}(f,[\mathcal{P}, c])}\right) \geq u_{a}\left(k_{P e_{b}(f,[\mathcal{P}, c])}\right)$, i.e., using the normalization $u_{i}\left(\delta_{0}\right)=0$ and $u_{i}\left(\delta_{M}\right)=1$ : $\Pi_{a}(f,[\mathcal{P}, c]) \geq \Pi_{b}(f,[\mathcal{P}, c])$.

$[(i i) \Rightarrow(i i i)]$

Let $(f,[\mathcal{P}, c]) \in \mathcal{A}_{B} \times \mathbb{S}$. Using the normalization $u_{i}\left(\delta_{0}\right)=0$ and $u_{i}\left(\delta_{M}\right)=1$, we get: $V_{i}(f,[\mathcal{P}, c])=\Pi_{i}(f,[\mathcal{P}, c])$, with $i \in\{a, b\}$. Assume that $\Pi_{a}(f,[\mathcal{P}, c]) \geq \Pi_{b}(f,[\mathcal{P}, c])$. This implies $V_{a}(f,[\mathcal{P}, c]) \geq V_{b}(f,[\mathcal{P}, c])$, for all $(f,[\mathcal{P}, c]) \in \mathcal{A}_{B} \times \mathbb{S}$. Therefore, using the representation given in Theorem $1,{ }^{7} \underset{p \in \mathcal{F}_{[\mathcal{P}, c]}^{a}}{\min } \int u \circ f d p \geq \min _{p \in \mathcal{F}_{[\mathcal{P}, c]}^{b}} \int u \circ f d p$. Assume $\mathcal{F}_{[\mathcal{P}, c]}^{a} \not \subset \mathcal{F}_{[\mathcal{P}, c]}^{b}$. Then, there

\footnotetext{
${ }^{7}$ Since $f \in \mathcal{A}_{B}$, we can, using the normalization above, restrict our attention to $u_{a}=u_{b}=u$.
} 
exists $p^{*} \in \mathcal{F}_{[\mathcal{P}, c]}^{a}$ such that $p^{*} \notin \mathcal{F}_{[\mathcal{P}, c]}^{b}$. Hence, using a separation argument, we know there exists a function $\phi: S \rightarrow \mathbb{R}$ such that $\int \phi d p^{*}<\min _{p \in \mathcal{F}_{[\mathcal{P}, c]}^{b}} \int \phi d p$. Since $S(\mathcal{P})$ is a finite set, there exist numbers $a>0$ and $b$, such that for all $s \in S(\mathcal{P}),(a \phi(s)+b \in u(Y))$. Then, for all $s \in S(\mathcal{P})$, there exits $y(s) \in Y_{B}$ such that $u(y(s))=a \phi(s)+b$. Define $f$ by $f(s)=y(s)$ for all $s \in S(\mathcal{P})$, and $f(s)=\delta_{0}$ for all $s \in S \backslash S(\mathcal{P})$. We then obtain: $\int u \circ f d p^{*}<\min _{p \in \mathcal{F}_{[\mathcal{P}, c]}^{b}} \int u \circ f d p$. Therefore, $\min _{p \in \mathcal{F}_{[\mathcal{P}, c]}^{a}} \int u \circ f d p<\min _{p \in \mathcal{F}_{[\mathcal{P}, c]}^{b}} \int u \circ f d p$, i.e., $V_{a}(f,[\mathcal{P}, c])=\Pi_{a}(f,[\mathcal{P}, c])<V_{b}(f,[\mathcal{P}, c])=V_{b}(f,[\mathcal{P}, c])$, which yields a contradiction.

$[(i i i) \Rightarrow(i)]$

Straightforward. 


\section{References}

Cohen, M., AND J.-Y. JAFFRAY (1980): "Rational behavior under complete ignorance," Econometrica, 48(5), 1281-1299.

EllsberG, D. (1961): "Risk, ambiguity, and the Savage axioms," Quarterly Journal of Economics, 75, 643-669.

Epstein, L. (1999): "A definition of uncertainty aversion," Review of Economic Studies, 66, $579-608$.

Epstein, L., AND J. Zhang (2001): "Subjective probabilities on subjectively unambiguous events," Econometrica, 69, 265-306.

Ghirardato, P., And M. MarinacCi (2002): "Ambiguity aversion made precise: a comparative foundation and some implications," Journal of Economic Theory, 102, 251-289.

GilboA, I., And D. Schmeidler (1989): "Maxmin expected utility with a non-unique prior," Journal of Mathematical Economics, 18, 141-153.

Hansen, L., and T. Sargent (2002): "Robust Control and Filetering for Macroeconomics," mimeo, Available at http://www.stanford.edu/sargent/robustness2.html.

Hansen, L., T. Sargent, G. Turmuhabmetova, and N. Williams (2001): "Robusteness and uncertainty aversion," mimeo.

JAFFrAY, J.-Y. (1989): "Linear utility for belief functions," Operations Research Letters, 8, $107-112$.

Klibanoff, P., M. Marinacci, and S. MukerJi (2003): "A Smooth Model of Decision Making Under Uncertainy," mimeo 11/2003, ICER.

Schmeidler, D. (1986): "Integral representation without additivity," Proceedings of the American Mathematical Society, 97(2), 255-261.

(1989): "Subjective probability and expected utility without additivity," Econometrica, $57(3), 571-587$.

SEgAL, U. (1987): "The Ellsberg paradox and risk: an anticipated utility approach," International Economic Review, 28, 175-202.

TAPKING, J. (2003): "Axioms for preferences revealing subjective uncertainty and uncertainty aversion," mimeo, European Central Bank.

von Neumann, J., And O. Morgenstern (1947): Theory of games and economic behavior. Princeton University Press.

WANG, T. (2003): "A class of multi-prior preferences," Discussion paper, University British Columbia. 